\title{
The dynamics of nutrient, toxic phytoplankton, nontoxic phytoplankton and zooplankton model
}

\author{
Hasnaa Fiesal Mohammed Hussien *, Raid Kamel Naji, Azhar Abbas Majeed \\ Department of Mathematic, College of Science, University of Baghdad, Baghdad-Iraq \\ *Corresponding author E-mail: hasnaafmh@gmail.com
}

\begin{abstract}
The objective of this paper is to study the dynamical behavior of an aquatic food web system. A mathematical model that includes nutrients, phytoplankton and zooplankton is proposed and analyzed. It is assumed that, the phytoplankton divided into two compartments namely toxic phytoplankton which produces a toxic substance as a defensive strategy against predation by zooplankton, and a nontoxic phytoplankton. All the feeding processes in this food web are formulating according to the Lotka-Volterra functional response. This model is represented mathematically by the set of nonlinear differential equations. The existence, uniqueness and boundedness of the solution of this model are investigated. The local and global stability conditions of all possible equilibrium points are established. The occurrence of local bifurcation and Hopf bifurcation are investigated. Finally, numerical simulation is used to study the global dynamics of this model.
\end{abstract}

Keywords: Aquatic Food Web; HOPF Bifurcation; Local Bifurcation; Stability Analysis.

\section{Introduction}

Nutrient-phytoplankton-zooplankton NPZ models have been used in oceanography for at least three decades, and are still a common research tool. The NPZ model incorporates one of the simplest sets of dynamics that usefully describe oceanic plankton dynamics [1]. Phytoplankton-zooplankton models have been studied by many authors [2-10]. Some type of phytoplankton produce toxin as a defensive strategy against the predation by zooplankton, these types are known as toxic phytoplankton. In [2], models of nutrient-plankton interaction with a toxic substance that inhibit either the growth rate of phytoplankton, zooplankton, or both trophic levels are proposed and studied. In [3], authors have dealt with a nutrient-plankton model in an aquatic environment in the context of phytoplankton bloom. Roy [4] has constructed a mathematical model for describing the interaction between a nontoxic and a toxic phytoplankton under a single nutrient. Saha and Bandyopadhyay [5] considered a toxin producing phytoplanktonzooplankton model. Since the phytoplankton is a base of all the aquatic food chain and food web systems and most of zooplankton organism depends directly on the phytoplankton in its feeding process. Therefore toxic substances released by toxic phytoplankton play an important role in this context see for example [11]. Phytoplankton organisms are the dominant primary producers in the pelagic environment. They convert inorganic materials into new organic compounds by the process of photosynthesis, starting there by most aquatic food webs [12]. Fan et al. [7] constructed a model to study a NPZ food chain ecosystem involving nontoxic phytoplankton. Rashid and Naji [13] proposed and analyzed NPZ food chain ecosystem model with a toxic phytoplankton. The objective of our model is to determine the interaction between (toxic, nontoxic) phytoplankton and zooplankton under single nutrient in food web ecosystem.

\section{Formulation of a mathematical model}

In this section, a food web system that contains nutrient, toxic phytoplankton, nontoxic phytoplankton and zooplankton is proposed and analyzed. It is assumed that the density of the nutrient at time $\mathrm{T}$ is denoted byN(T), the density of toxic phytoplankton at time $\mathrm{T}$ represents by $\mathrm{P}_{1}(\mathrm{~T})$, while $\mathrm{P}_{2}(\mathrm{~T})$ represents the density of the nontoxic phytoplankton at timeT. Finally the density of the zooplankton at time $\mathrm{T}$ denote $\mathrm{by}_{3}(\mathrm{~T})$. Now, in order to formulate the interaction in the above system among these species mathematically the following assumptions are obtained:

1) There is a constant concentration of nutrient inter to the system $\mathrm{N}_{0}>0$ with constant rate of dilution $\mathrm{D}>0$. The nutrient uptakes by toxic phytoplankton $\mathrm{P}_{1}$ and nontoxic phytoplankton $\mathrm{P}_{2}$ according to Lotka-Volterra type of functional response with consumption rates $\alpha_{1}>0$ and $\alpha_{2}>0$ respectively, and conversion rates $\mathrm{k}_{1}>0$ and $\mathrm{k}_{2}>0$ respectively. On the other hand a portion of the dead toxic phytoplankton $\mathrm{P}_{1}$, non-toxic phytoplankton $\mathrm{P}_{2}$ and zooplankton $\mathrm{P}_{3}$ return to the nutrient due to the decomposition operation with rates $0<\mathrm{m}_{1}<1,0<\mathrm{m}_{2}<1$ and $0<\mathrm{m}_{3}<1$ respectively.

2) In the absence of nutrient the toxic phytoplankton $P_{1}$ and the non-toxic phytoplankton $\mathrm{P}_{2}$ decay exponentially due to dilution and natural death rates $\varepsilon_{1}>0$ and $\varepsilon_{2}>0$ respectively. Further decay facing the toxic phytoplankton $\mathrm{P}_{1}$ and the non-phytoplankton $\mathrm{P}_{2}$ due to the feeding process by zooplankton $\mathrm{P}_{3}$.

3) The zooplankton feeds on the toxic phytoplankton $P_{1}$ and the non-toxic phytoplankton $\mathrm{P}_{2}$ according to Lotka-Volterra type of functional response with consumption rates $\beta_{1}>0$ and $\beta_{2}>0$ respectively, and conversion rates $\mathrm{k}_{3}>0$ and $\mathrm{k}_{4}>0$ respectively. Further it is assumed that the zooplankton affected by the toxin produced by the toxic phytoplank- 
ton $\mathrm{P}_{1}$ during the predation process, with $\theta$ which stand for the liberation rate of toxin substance, $\delta$ the maximum zooplankton in gestation rate for the toxic substance.

Consequently, the dynamics of the above system can be formulated mathematical by the following set of equations: An easy way to comply with the paper formatting requirements is to use this document as a template and simply type your text into it.

$$
\begin{aligned}
& \frac{\mathrm{dN}}{\mathrm{dT}}=\mathrm{D}\left(\mathrm{N}_{0}-\mathrm{N}\right)-\alpha_{1} \mathrm{NP}_{1}-\alpha_{2} \mathrm{NP}_{2}+\mathrm{m}_{1} \varepsilon_{1} \mathrm{P}_{1}+\mathrm{m}_{2} \varepsilon_{2} \mathrm{P}_{2}+\mathrm{m}_{3} \varepsilon_{3} \mathrm{P}_{3} \\
& \frac{\mathrm{dP}}{\mathrm{dT}}=\mathrm{k}_{1} \alpha_{1} \mathrm{NP}_{1}-\left(\mathrm{D}+\varepsilon_{1}\right) \mathrm{P}_{1}-\beta_{1} \mathrm{P}_{1} \mathrm{P}_{3}
\end{aligned}
$$

$\frac{d P_{2}}{d T}=k_{2} \alpha_{2} N_{2}-\left(D+\varepsilon_{2}\right) P_{2}-\beta_{2} P_{2} P_{3}$

$\frac{d P_{3}}{d T}=k_{3} \beta_{1} P_{1} P_{3}+k_{4} \beta_{2} P_{2} P_{3}-\left(D+\varepsilon_{3}\right) P_{3}-\theta \delta P_{1} P_{3}$

Note that the above proposed model has eighteen parameters in all, which make the analysis difficult. So, in order to simplify the system, the number of parameters is reduced by using the following dimensionless variables and parameters:

$$
\begin{aligned}
& \mathrm{t}=\mathrm{DT}, \mathrm{x}=\frac{\mathrm{N}}{\mathrm{N}_{0}}, \mathrm{y}=\frac{\alpha_{1}}{\mathrm{D}} \mathrm{P}_{1}, \mathrm{z}=\frac{\alpha_{2}}{\mathrm{D}} \mathrm{P}_{2}, \mathrm{w}=\frac{\mathrm{m}_{3} \varepsilon_{3}}{\mathrm{DN}_{0}} \mathrm{P}_{3}, \\
& \mathrm{u}_{1}=\frac{\mathrm{m}_{1} \varepsilon_{1}}{\alpha_{1} \mathrm{~N}_{0}}, \mathrm{u}_{2}=\frac{\mathrm{m}_{2} \varepsilon_{2}}{\alpha_{2} \mathrm{~N}_{0}}, \mathrm{u}_{3}=\frac{\mathrm{k}_{1} \alpha_{1} \mathrm{~N}_{0}}{\mathrm{D}}, \mathrm{u}_{4}=\frac{\left(\mathrm{D}+\varepsilon_{1}\right)}{\mathrm{D}}, \\
& \mathrm{u}_{5}=\frac{\beta_{1} \mathrm{~N}_{0}}{\mathrm{~m}_{3} \varepsilon_{3}}, \mathrm{u}_{6}=\frac{\mathrm{k}_{2} \alpha_{2} \mathrm{~N}_{0}}{\mathrm{D}}, \mathrm{u}_{7}=\frac{\left(\mathrm{D}+\varepsilon_{2}\right)}{\mathrm{D}} \mathrm{u}_{8}=\frac{\beta_{2} \mathrm{~N}_{0}}{\mathrm{~m}_{3} \varepsilon_{3}}, \\
& \mathrm{u}_{9}=\frac{\mathrm{k}_{3} \beta_{1}}{\alpha_{1}}, \mathrm{u}_{10}=\frac{\mathrm{k}_{4} \beta_{2}}{\alpha_{2}}, \mathrm{u}_{11}=\frac{\left(\mathrm{D}+\varepsilon_{3}\right)}{\mathrm{D}}, \mathrm{u}_{12}=\frac{\theta \delta}{\alpha_{1}}
\end{aligned}
$$

Then the non-dimensional form of system (1) can be written as:

$$
\begin{aligned}
& \frac{d x}{d t}=1-x-x y-x z+u_{1} y+u_{2} z+w=F_{1}(x, y, z, w) \\
& \frac{d y}{d t}=u_{3} x y-u_{4} y-u_{5} y w=F_{2}(x, y, z, w) \\
& \frac{d z}{d t}=u_{6} x z-u_{7} z-u_{8} z w=F_{3}(x, y, z, w) \\
& \frac{d w}{d t}=s_{1} y w+u_{10} z w-u_{11} w=F_{4}(x, y, z, w)
\end{aligned}
$$

Where $s_{1}=u_{9}-u_{12}$, with initial condition $x(0) \geq 0, y(0) \geq$ $0, \mathrm{z}(0) \geq 0$ and $\mathrm{w}(0) \geq 0$. It is observed that the number of parameters have been reduced from eighteen in the system (1) to twelve in the system (2). Obviously the interaction functions of the system (2) are continuous and have continuous partial derivatives on the four dimensional space

$$
\mathrm{R}_{+}^{4}=\left\{(\mathrm{x}, \mathrm{y}, \mathrm{z}, \mathrm{w}) \in \mathrm{R}^{4}: \mathrm{x}(0) \geq 0, \mathrm{y}(0) \geq 0, \mathrm{z}(0) \geq 0, \mathrm{w}(0) \geq 0\right\}
$$

Therefore these functions are Lipschitizion on $\mathrm{R}_{+}^{4}$, and hence the solution of the system (2) exists and is unique. Moreover, the boundedness of the solution of the system (2) in $\mathrm{R}_{+}^{4}$ is established in the following theorem.

Theorem 1: All the solutions of system (2) which initiate in $R_{+}^{4}$ are uniformly bounded as $t$ goes to $\infty$.

Proof: Let $(\mathrm{x}(\mathrm{t}), \mathrm{y}(\mathrm{t}), \mathrm{z}(\mathrm{t}), \mathrm{w}(\mathrm{t}))$ be any solution of the system (2) with non-negative initial condition $\left(\mathrm{x}_{0}, \mathrm{y}_{0}, \mathrm{z}_{0}, \mathrm{w}_{0}\right) \in \mathrm{R}_{+}^{4}$.

Define the function: $\mathrm{M}(\mathrm{t})=\mathrm{x}(\mathrm{t})+\mathrm{y}(\mathrm{t})+\mathrm{z}(\mathrm{t})+\mathrm{w}(\mathrm{t})$, and then take the time derivative of $\mathrm{M}(\mathrm{t})$ along the solution of the system (2) we get:

$\frac{d M}{d t} \leq 1-x-\left(u_{4}-u_{1}\right) y-\left(u_{7}-u_{2}\right) z-\left(u_{11}-1\right) w$
Then $\frac{d M}{d t}+s M \leq 1$, where $s=\min \left\{1, u_{4}-u_{1}, u_{7}-u_{2}, u_{11}-\right.$ $1\}$.

Now, by using the comparison theorem on the differential inequality for the initial valueM $(0)=\mathrm{M}_{0}$, we get:

$M(t) \leq \frac{1}{s}+\left(M_{0}-\frac{1}{s}\right) e^{-s t}$

Thus, $\lim _{\mathrm{t} \rightarrow \infty} \mathrm{M}(\mathrm{t}) \leq \frac{1}{\mathrm{~s}}$, and hence $0 \leq \mathrm{M}(\mathrm{t}) \leq \frac{1}{\mathrm{~s}}, \forall \mathrm{t}>0$. So, all the solutions of system (2) are uniformly bounded as $t \rightarrow \infty$.

\section{Existence of equilibrium points}

It is observed that, system (2) has at most seven biologically feasible equilibrium points, namelyE $\mathrm{E}_{\mathrm{i}}, \mathrm{i}=0,1,2,3,4,5,6$. The existence conditions for each of these equilibrium points are discussed in the following:

- The single species equilibrium point $\mathrm{E}_{0}=(1,0,0,0)$ always exists.

- The first planar equilibrium point $\mathrm{E}_{1}=\left(\mathrm{x}_{1}, \mathrm{y}_{1}, 0,0\right)$, where:

$\mathrm{x}_{1}=\frac{\mathrm{u}_{4}}{\mathrm{u}_{3}}$ and $\mathrm{y}_{1}=\frac{\mathrm{u}_{4}-\mathrm{u}_{3}}{\mathrm{u}_{1} \mathrm{u}_{3}-\mathrm{u}_{4}}$

Exists uniquely in Int. $R_{+}^{2}$ (interior of $R_{+}^{2}$ ) of $x y$-plane under the following necessary and sufficient condition:

$\mathrm{u}_{1} \mathrm{u}_{3}<\mathrm{u}_{4}<\mathrm{u}_{3}$ or $\mathrm{u}_{1} \mathrm{u}_{3}>\mathrm{u}_{4}>\mathrm{u}_{3}$

- The second planar equilibrium point $\mathrm{E}_{2}=\left(\mathrm{x}_{2}, 0, \mathrm{z}_{2}, 0\right)$, where:

$\mathrm{x}_{2}=\frac{\mathrm{u}_{7}}{\mathrm{u}_{6}}$ and $\mathrm{z}_{2}=\frac{\mathrm{u}_{7}-\mathrm{u}_{6}}{\mathrm{u}_{2} \mathrm{u}_{6}-\mathrm{u}_{7}}$

Exists uniquely in Int. $\mathrm{R}_{+}^{2}$ of $\mathrm{xz}-$ plane under the following necessary and sufficient condition:

$\mathrm{u}_{2} \mathrm{u}_{6}<\mathrm{u}_{7}<\mathrm{u}_{6}$ or $\mathrm{u}_{2} \mathrm{u}_{6}>\mathrm{u}_{7}>\mathrm{u}_{6}$

$\left.0, \mathrm{w}_{3}\right)$, where:

The first $3 D$ boundary equilibrium point $E_{3}=\left(x_{3}, y_{3}\right.$,

$\mathrm{x}_{3}=\frac{\mathrm{u}_{4} \mathrm{~s}_{1}-\mathrm{u}_{5}\left(\mathrm{u}_{1} \mathrm{u}_{11}+\mathrm{s}_{1}\right)}{\mathrm{u}_{3} \mathrm{~s}_{1}-\mathrm{u}_{5}\left(\mathrm{u}_{11}+\mathrm{s}_{1}\right)}, \mathrm{y}_{3}=\frac{\mathrm{u}_{11}}{\mathrm{~s}_{1}}$,

$\mathrm{w}_{3}=\frac{\mathrm{u}_{4}\left(\mathrm{u}_{11}+\mathrm{s}_{1}\right)-\mathrm{u}_{3}\left(\mathrm{u}_{1} \mathrm{u}_{11}+\mathrm{s}_{1}\right)}{\mathrm{u}_{3} \mathrm{~s}_{1}-\mathrm{u}_{5}\left(\mathrm{u}_{11}+\mathrm{s}_{1}\right)}$

Exists uniquely in Int. $\mathrm{R}_{+}^{3}$ of xyw - space under the following necessary and sufficient conditions:

$\mathrm{s}_{1}>0$

With

$\left.\begin{array}{c}\mathrm{u}_{4}\left(\mathrm{u}_{11+} \mathrm{s}_{1}\right)>\mathrm{u}_{3}\left(\mathrm{u}_{1} \mathrm{u}_{11}+\mathrm{s}_{1}\right) \\ \mathrm{u}_{4} \mathrm{~s}_{1}>\mathrm{u}_{5}\left(\mathrm{u}_{1} \mathrm{u}_{11}+\mathrm{s}_{1}\right) \\ \mathrm{u}_{3} \mathrm{~s}_{1}>\mathrm{u}_{5}\left(\mathrm{u}_{11}+\mathrm{s}_{1}\right)\end{array}\right\}$

Or

$\left.\begin{array}{c}\mathrm{u}_{4}\left(\mathrm{u}_{11+} \mathrm{s}_{1}\right)<\mathrm{u}_{3}\left(\mathrm{u}_{1} \mathrm{u}_{11}+\mathrm{s}_{1}\right) \\ \mathrm{u}_{4} \mathrm{~s}_{1}<\mathrm{u}_{5}\left(\mathrm{u}_{1} \mathrm{u}_{11}+\mathrm{s}_{1}\right) \\ \mathrm{u}_{3} \mathrm{~s}_{1}<\mathrm{u}_{5}\left(\mathrm{u}_{11}+\mathrm{s}_{1}\right)\end{array}\right\}$

- $\quad$ The second 3D boundary equilibrium point $\mathrm{E}_{4}=\left(\mathrm{x}_{4}\right.$, $0, \mathrm{z}_{4}, \mathrm{w}_{4}$ ), where: 
$\mathrm{x}_{4}=\frac{\mathrm{u}_{7} \mathrm{u}_{10}-\mathrm{u}_{8}\left(\mathrm{u}_{2} \mathrm{u}_{11}+\mathrm{u}_{10}\right)}{\mathrm{u}_{6} \mathrm{u}_{10}-\mathrm{u}_{8}\left(\mathrm{u}_{10}+\mathrm{u}_{11}\right)}, \mathrm{z}_{4}=\frac{\mathrm{u}_{11}}{\mathrm{u}_{10}}$

$\mathrm{w}_{4}=\frac{\mathrm{u}_{7}\left(\mathrm{u}_{10}+\mathrm{u}_{11}\right)-\mathrm{u}_{6}\left(\mathrm{u}_{2} \mathrm{u}_{11}+\mathrm{u}_{10}\right)}{\mathrm{u}_{6} \mathrm{u}_{10}-\mathrm{u}_{8}\left(\mathrm{u}_{10}+\mathrm{u}_{11}\right)}$

Exists uniquely in Int. $\mathrm{R}_{+}^{3}$ of xzw - space under the following necessary and sufficient conditions:

$$
\left.\begin{array}{c}
\mathrm{u}_{7}\left(\mathrm{u}_{10}+\mathrm{u}_{11}\right)>\mathrm{u}_{6}\left(\mathrm{u}_{2} \mathrm{u}_{11}+\mathrm{u}_{10}\right) \\
\mathrm{u}_{7} \mathrm{u}_{10}>\mathrm{u}_{8}\left(\mathrm{u}_{2} \mathrm{u}_{11}+\mathrm{u}_{10}\right) \\
\mathrm{u}_{6} \mathrm{u}_{10}>\mathrm{u}_{8}\left(\mathrm{u}_{10}+\mathrm{u}_{11}\right)
\end{array}\right\}
$$

$\mathrm{Or}$

$$
\left.\begin{array}{c}
\mathrm{u}_{7}\left(\mathrm{u}_{10}+\mathrm{u}_{11}\right)<\mathrm{u}_{6}\left(\mathrm{u}_{2} \mathrm{u}_{11}+\mathrm{u}_{10}\right) \\
\mathrm{u}_{7} \mathrm{u}_{10}<\mathrm{u}_{8}\left(\mathrm{u}_{2} \mathrm{u}_{11}+\mathrm{u}_{10}\right) \\
\mathrm{u}_{6} \mathrm{u}_{10}<\mathrm{u}_{8}\left(\mathrm{u}_{10}+\mathrm{u}_{11}\right)
\end{array}\right\}
$$

- The third 3D boundary equilibrium point $\mathrm{E}_{5}=$ as: $\left(\mathrm{x}_{5}, \mathrm{y}_{5}, \mathrm{z}_{5}, 0\right)$, where:

$\mathrm{x}_{5}=\frac{\mathrm{u}_{4}}{\mathrm{u}_{3}}, \mathrm{y}_{5}=\frac{\mathrm{u}_{4}-\mathrm{u}_{3}+\left(\mathrm{u}_{4}-\mathrm{u}_{2} \mathrm{u}_{3}\right) \mathrm{z}_{5}}{\mathrm{u}_{1} \mathrm{u}_{3}-\mathrm{u}_{4}}$,

$\mathrm{z}_{5}=\frac{\mathrm{u}_{4}-\mathrm{u}_{3}+\left(\mathrm{u}_{4}-\mathrm{u}_{1} \mathrm{u}_{3}\right) \mathrm{y}_{5}}{\mathrm{u}_{2} \mathrm{u}_{3}-\mathrm{u}_{4}}$

Exists uniquely in Int. $\mathrm{R}_{+}^{3}$ of $\mathrm{xyz}$ - space under the following necessary and sufficient conditions:

$\mathrm{u}_{4} \mathrm{u}_{6}=\mathrm{u}_{3} \mathrm{u}_{7}$

With

$$
\left.\begin{array}{l}
\mathrm{u}_{4}\left(1+\mathrm{z}_{5}\right)>\mathrm{u}_{3}\left(1+\mathrm{u}_{2} \mathrm{z}_{5}\right), \text { and } \mathrm{u}_{1} \mathrm{u}_{3}>\mathrm{u}_{4} \\
\mathrm{u}_{4}\left(1+\mathrm{y}_{5}\right)>\mathrm{u}_{3}\left(1+\mathrm{u}_{1} \mathrm{y}_{5}\right), \text { and } \mathrm{u}_{2} \mathrm{u}_{3}<\mathrm{u}_{4}
\end{array}\right\}
$$

Or

$\mathrm{u}_{4}\left(1+\mathrm{z}_{5}\right)<\mathrm{u}_{3}\left(1+\mathrm{u}_{2} \mathrm{z}_{5}\right)$, and $\left.\mathrm{u}_{1} \mathrm{u}_{3}<\mathrm{u}_{4}\right)$

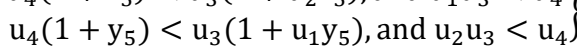

Finally,

- The positive (coexistence) equilibrium point $\mathrm{E}_{6}=$ $\left(\mathrm{x}_{6}, \mathrm{y}_{6}, \mathrm{z}_{6}, \mathrm{w}_{6}\right)$, where:

$\mathrm{x}_{6}=\frac{\mathrm{s}_{2}}{\mathrm{~s}_{4}}, \mathrm{y}_{6}=\frac{\mathrm{s}_{4}\left(\mathrm{u}_{2} \mathrm{u}_{11}+\mathrm{u}_{10}\right)+\mathrm{u}_{10} \mathrm{~s}_{3}-\mathrm{s}_{2}\left(\mathrm{u}_{10}+\mathrm{u}_{11}\right)}{\mathrm{u}_{2} \mathrm{~s}_{1} \mathrm{~s}_{4}+\mathrm{u}_{10} \mathrm{~s}_{2}-\mathrm{s}_{1} \mathrm{~s}_{2}-\mathrm{u}_{1} \mathrm{u}_{10} \mathrm{~s}_{4}}$

$z_{6}=\frac{s_{2}\left(u_{11}+s_{1}\right)-u_{1} u_{11} s_{4}-s_{1}\left(s_{3}+s_{4}\right)}{u_{2} s_{1} s_{4}+u_{10} s_{2}-s_{1} s_{2}-u_{1} u_{10} s_{4}}, w_{6}=\frac{s_{3}}{s_{4}}$

Here $\mathrm{s}_{2}=\mathrm{u}_{5} \mathrm{u}_{7}-\mathrm{u}_{4} \mathrm{u}_{8}, \mathrm{~s}_{3}=\mathrm{u}_{3} \mathrm{u}_{7}-\mathrm{u}_{4} \mathrm{u}_{6}, \mathrm{~s}_{4}=\mathrm{u}_{5} \mathrm{u}_{6}-\mathrm{u}_{3} \mathrm{u}_{8}$ exists uniquely in Int. $\mathrm{R}_{+}^{4}$ under the following necessary and sufficient conditions:

$\mathrm{u}_{11}>\mathrm{u}_{10} \mathrm{z}_{6}$

With

$\mathrm{s}_{2}>0, \mathrm{~s}_{3}>0$ and $\mathrm{s}_{4}>0$

or

$\mathrm{s}_{2}<0, \mathrm{~s}_{3}<0$ and $\left.\mathrm{s}_{4}<0\right\}$

With

$\left.\begin{array}{c}s_{2}\left(u_{11}+s_{1}\right)>u_{1} u_{11} s_{4}+s_{1}\left(s_{3}+s_{4}\right) \\ u_{2} s_{1} s_{4}+u_{10} s_{2}>s_{1} s_{2}+u_{1} u_{10} s_{4}\end{array}\right\}$

Or

$$
\left.\begin{array}{c}
s_{2}\left(u_{11}+s_{1}\right)<u_{1} u_{11} s_{4}+s_{1}\left(s_{3}+s_{4}\right) \\
u_{2} s_{1} s_{4}+u_{10} s_{2}<s_{1} s_{2}+u_{1} u_{10} s_{4}
\end{array}\right\}
$$

\section{Local stability analysis}

In this section, the local stability analysis of system (2) around each of the above equilibrium points is discussed through computing the Jacobian matrix $J(x, y, z, w)$ of system (2) at each of them. The general Jacobain matrix of system (2) can be written as follows:

$J=\left[\begin{array}{cccc}-(1+y+z) & u_{1}-x & u_{2}-x & 1 \\ u_{3} y & u_{3} x-u_{4}-u_{5} w & 0 & -u_{5} y \\ u_{6} z & 0 & u_{6} x-u_{7}-u_{8} w & -u_{8} z \\ 0 & s_{1} w & u_{10} w & s_{1} y-u_{10} z-u_{11}\end{array}\right]$

- The Jacobian matrix of system (2) at $\mathrm{E}_{0}$ can be written

$\mathrm{J}_{0}=\left[\begin{array}{cccc}-1 & \mathrm{u}_{1}-1 & \mathrm{u}_{2}-1 & 1 \\ 0 & \mathrm{u}_{3}-\mathrm{u}_{4} & 0 & 0 \\ 0 & 0 & \mathrm{u}_{6}-\mathrm{u}_{7} & 0 \\ 0 & 0 & 0 & -\mathrm{u}_{11}\end{array}\right]$

Clearly, $\mathrm{J}_{0}$ has the following eigenvalues:

$\lambda_{0 \mathrm{x}}=-1, \lambda_{0 \mathrm{y}}=\mathrm{u}_{3}-\mathrm{u}_{4}, \lambda_{0 \mathrm{z}}=\mathrm{u}_{6}-\mathrm{u}_{7}, \lambda_{0 \mathrm{w}}=-\mathrm{u}_{11}$

Therefore all the eigenvalues have negative real parts and hence the equilibrium point $\mathrm{E}_{0}$ is locally asymptotically stable provided that

$\mathrm{u}_{4}>\mathrm{u}_{3}$

$\mathrm{u}_{7}>\mathrm{u}_{6}$

Otherwise it will be saddle point.

- The Jacobian matrix of system (2) at $\mathrm{E}_{1}$ can be written as:

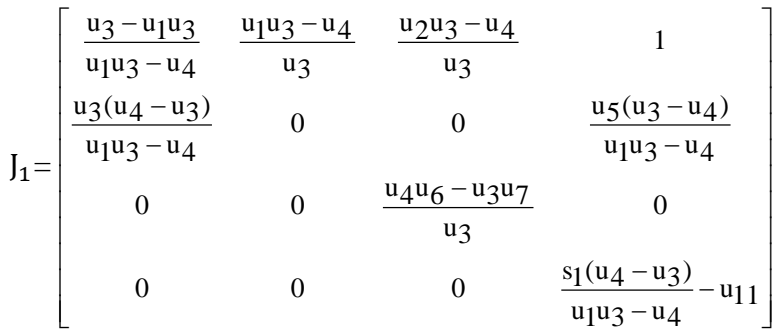

The characteristic equation of this Jacobian matrix is given by:

$\left\{\left(\frac{\mathrm{s}_{1}\left(\mathrm{u}_{4}-\mathrm{u}_{3}\right)}{\mathrm{u}_{1} \mathrm{u}_{3}-\mathrm{u}_{4}}-\mathrm{u}_{11}-\lambda_{1 \mathrm{w}}\right)\left(\frac{\mathrm{u}_{4} \mathrm{u}_{6}-\mathrm{u}_{3} \mathrm{u}_{7}}{\mathrm{u}_{3}}-\lambda_{1 \mathrm{z}}\right)\right.$

$\left.\left[\lambda_{1}^{2}-\frac{\mathrm{u}_{3}-\mathrm{u}_{1} \mathrm{u}_{3}}{\mathrm{u}_{1} \mathrm{u}_{3}-\mathrm{u}_{4}} \lambda_{1}+\left(\mathrm{u}_{3}-\mathrm{u}_{4}\right)\right]\right\}=0$

Therefore,

$\lambda_{1 \mathrm{x}}, \lambda_{1 \mathrm{y}}=\frac{\mathrm{u}_{3}-\mathrm{u}_{1} \mathrm{u}_{3}}{2\left(\mathrm{u}_{1} \mathrm{u}_{3}-\mathrm{u}_{4}\right)} \pm \frac{1}{2} \sqrt{\left(\frac{\mathrm{u}_{3}-\mathrm{u}_{1} \mathrm{u}_{3}}{\mathrm{u}_{1} \mathrm{u}_{3}-\mathrm{u}_{4}}\right)^{2}-4\left(\mathrm{u}_{3}-\mathrm{u}_{4}\right)}$,

$\lambda_{1 \mathrm{z}}=\frac{\mathrm{u}_{4} \mathrm{u}_{6}-\mathrm{u}_{3} \mathrm{u}_{7}}{\mathrm{u}_{3}}, \lambda_{1 \mathrm{w}}=\frac{\mathrm{s}_{1}\left(\mathrm{u}_{4}-\mathrm{u}_{3}\right)}{\mathrm{u}_{1} \mathrm{u}_{3}-\mathrm{u}_{4}}-\mathrm{u}_{11}$ 
Then all the eigenvalues have negative real parts and hence the equilibrium point $E_{1}$ is locally asymptotically stable, if the existence condition ( $3 b$ ) along with the following conditions satisfies:

$u_{3} u_{7}>u_{4} u_{6}$

$\mathrm{u}_{11}>\frac{\mathrm{s}_{1}\left(\mathrm{u}_{4}-\mathrm{u}_{3}\right)}{\mathrm{u}_{1} \mathrm{u}_{3}-\mathrm{u}_{4}}$

- The Jacobian matrix of system (2) at $\mathrm{E}_{2}$ can be written as:

$$
\mathrm{J}_{2}=\left[\begin{array}{cccc}
\frac{\mathrm{u}_{6}-\mathrm{u}_{2} \mathrm{u}_{6}}{\mathrm{u}_{2} \mathrm{u}_{6}-\mathrm{u}_{7}} & \frac{\mathrm{u}_{1} \mathrm{u}_{6}-\mathrm{u}_{7}}{\mathrm{u}_{6}} & \frac{\mathrm{u}_{2} \mathrm{u}_{6}-\mathrm{u}_{7}}{\mathrm{u}_{6}} & 1 \\
0 & \frac{\mathrm{u}_{3} \mathrm{u}_{7}-\mathrm{u}_{4} \mathrm{u}_{6}}{\mathrm{u}_{6}} & 0 & 0 \\
\frac{\mathrm{u}_{6}\left(\mathrm{u}_{7}-\mathrm{u}_{6}\right)}{\mathrm{u}_{2} \mathrm{u}_{6}-\mathrm{u}_{7}} & 0 & 0 & \frac{\mathrm{u}_{8}\left(\mathrm{u}_{6}-\mathrm{u}_{7}\right)}{\mathrm{u}_{2} \mathrm{u}_{6}-\mathrm{u}_{7}} \\
0 & 0 & 0 & \frac{\mathrm{u}_{10}\left(\mathrm{u}_{7}-\mathrm{u}_{6}\right)}{\mathrm{u}_{2} \mathrm{u}_{6}-\mathrm{u}_{7}}-\mathrm{u}_{11}
\end{array}\right]
$$

The characteristic equation of this Jacobian matrix is given by:

$$
\begin{aligned}
& \left\{\left(\frac{\mathrm{u}_{10}\left(\mathrm{u}_{7}-\mathrm{u}_{6}\right)}{\mathrm{u}_{2} \mathrm{u}_{6}-\mathrm{u}_{7}}-\mathrm{u}_{11}-\lambda_{2 \mathrm{w}}\right)\left(\frac{\mathrm{u}_{3} \mathrm{u}_{7}-\mathrm{u}_{4} \mathrm{u}_{6}}{\mathrm{u}_{6}}-\lambda_{2 \mathrm{y}}\right)\right. \\
& \left.\left[\lambda_{2}^{2}-\frac{\mathrm{u}_{6}-\mathrm{u}_{2} \mathrm{u}_{6}}{\mathrm{u}_{2} \mathrm{u}_{6}-\mathrm{u}_{7}} \lambda_{2}+\left(\mathrm{u}_{6}-\mathrm{u}_{7}\right)\right]\right\}=0
\end{aligned}
$$

Therefore,

$$
\begin{aligned}
\lambda_{2 \mathrm{x}}, \lambda_{2 \mathrm{z}}=\frac{\mathrm{u}_{6}-\mathrm{u}_{2} \mathrm{u}_{6}}{2\left(\mathrm{u}_{2} \mathrm{u}_{6}-\mathrm{u}_{7}\right)} \pm \frac{1}{2} \sqrt{\left(\frac{\mathrm{u}_{6}-\mathrm{u}_{2} \mathrm{u}_{6}}{\mathrm{u}_{2} \mathrm{u}_{6}-\mathrm{u}_{7}}\right)^{2}-4\left(\mathrm{u}_{6}-\mathrm{u}_{7}\right)}, \\
\lambda_{2 \mathrm{y}}=\frac{\mathrm{u}_{3} \mathrm{u}_{7}-\mathrm{u}_{4} \mathrm{u}_{6}}{\mathrm{u}_{6}}, \lambda_{2 \mathrm{w}}=\frac{\mathrm{u}_{10}\left(\mathrm{u}_{7}-\mathrm{u}_{6}\right)}{\mathrm{u}_{2} \mathrm{u}_{6}-\mathrm{u}_{7}}-\mathrm{u}_{11}
\end{aligned}
$$

Then all the eigenvalues have negative real parts and hence the equilibrium point $E_{2}$ is locally asymptotically stable if the existence condition (4b) along with the following conditions satisfies:

$\mathrm{u}_{4} \mathrm{u}_{6}>\mathrm{u}_{3} \mathrm{u}_{7}$

$u_{11}>\frac{u_{10}\left(u_{7}-u_{6}\right)}{u_{2} u_{6}-u_{7}}$

- The Jacobian matrix of system (2) at $E_{3}$ can be written as:

$\mathrm{J}_{3}=\left[\begin{array}{cccc}-\left(1+\mathrm{y}_{3}\right) & \mathrm{u}_{1}-\mathrm{x}_{3} & \mathrm{u}_{2}-\mathrm{x}_{3} & 1 \\ \mathrm{u}_{3} \mathrm{y}_{3} & 0 & 0 & -\mathrm{u}_{5} \mathrm{y}_{3} \\ 0 & 0 & \mathrm{u}_{6} \mathrm{x}_{3}-\mathrm{u}_{7}-\mathrm{u}_{8} \mathrm{w}_{3} & 0 \\ 0 & \mathrm{~s}_{1} \mathrm{w}_{3} & \mathrm{u}_{10} \mathrm{w}_{3} & 0\end{array}\right]=\left(\mathrm{a}_{\mathrm{ij}}\right)_{4 \times 4}$

The characteristic equation of $\mathrm{J}_{3}$ can be written as:

$$
\left(\mathrm{a}_{33}-\lambda_{3 \mathrm{z}}\right)\left[\lambda_{3}^{3}+\mathrm{A}_{1} \lambda_{3}^{2}+\mathrm{A}_{2} \lambda_{3}+\mathrm{A}_{3}\right]=0
$$

Here

$\mathrm{A}_{1}=-\mathrm{a}_{11}, \mathrm{~A}_{2}=-\left(\mathrm{a}_{24} \mathrm{a}_{42}+\mathrm{a}_{12} \mathrm{a}_{21}\right), \mathrm{A}_{3}=\mathrm{a}_{42}\left(\mathrm{a}_{11} \mathrm{a}_{24}-\mathrm{a}_{21} \mathrm{a}_{14}\right)$

Now, it is easy to verify that:
$\Delta=\mathrm{A}_{1} \mathrm{~A}_{2}-\mathrm{A}_{3}=\mathrm{a}_{21}\left(\mathrm{a}_{11} \mathrm{a}_{12}+\mathrm{a}_{14} \mathrm{a}_{42}\right)$.

Clearly, the eigenvalue $\lambda_{3 z}$ in z-direction has negative real part if and only if the following condition holds:

$\mathrm{u}_{6} \mathrm{x}_{3}<\mathrm{u}_{7}+\mathrm{u}_{8} \mathrm{w}_{3}$

However, according to existence condition (5c), it is observed that $A_{i}>0, \forall i=1,3$, further $\Delta>0$ if and only if:

$\mathrm{u}_{3}<\mathrm{u}_{5}\left(1+\mathrm{y}_{3}\right)$

$\mathrm{u}_{1}<\mathrm{x}_{3}$

So, according to Routh-Hurwitz criterion the roots of the third degree polynomial in the characteristic equation have negative real parts and hence the equilibrium point $\mathrm{E}_{3}$ is locally asymptotically stable. as:

The Jacobian matrix of system (2) at $\mathrm{E}_{4}$ can be written

$\mathrm{J}_{4}=\left[\begin{array}{cccc}-\left(1+\mathrm{z}_{4}\right) & \mathrm{u}_{1}-\mathrm{x}_{4} & \mathrm{u}_{2}-\mathrm{x}_{4} & 1 \\ 0 & \mathrm{u}_{3} \mathrm{x}_{4}-\mathrm{u}_{4}-\mathrm{u}_{5} \mathrm{w}_{4} & 0 & 0 \\ \mathrm{u}_{6} \mathrm{z}_{4} & 0 & 0 & -\mathrm{u}_{8} \mathrm{z}_{4} \\ 0 & \mathrm{~s}_{1} \mathrm{w}_{4} & \mathrm{u}_{10} \mathrm{w}_{4} & \mathrm{u}_{10} \mathrm{z}_{4}-\mathrm{u}_{11}\end{array}\right]=\left(\mathrm{b}_{\mathrm{ij}}\right)_{4 \times 4}$

The characteristic equation of $\mathrm{J}_{4}$ can be written as:

$\left(\mathrm{b}_{22}-\lambda_{4 \mathrm{y}}\right)\left[\lambda_{4}^{3}+\mathrm{B}_{1} \lambda_{4}^{2}+\mathrm{B}_{2} \lambda_{4}+\mathrm{B}_{3}\right]=0$

Here

$\mathrm{B}_{1}=-\mathrm{b}_{11}, \mathrm{~B}_{2}=-\left(\mathrm{b}_{43} \mathrm{~b}_{34}+\mathrm{b}_{13} \mathrm{~b}_{31}\right), \mathrm{B}_{3}=\mathrm{b}_{43}\left(\mathrm{~b}_{11} \mathrm{~b}_{34}-\mathrm{b}_{31} \mathrm{~b}_{14}\right)$

Further, it is easy to verify that:

$\Delta=\mathrm{B}_{1} \mathrm{~B}_{2}-\mathrm{B}_{3}=\mathrm{b}_{31}\left(\mathrm{~b}_{11} \mathrm{~b}_{13}+\mathrm{b}_{14} \mathrm{~b}_{43}\right)$.

Clearly, the eigenvalue $\lambda_{4 y}$ in y-direction has negative real part if and only if the following condition holds:

$\mathrm{u}_{3} \mathrm{x}_{4}<\mathrm{u}_{4}+\mathrm{u}_{5} \mathrm{w}_{4}$

However, according to existence condition (6b), we obtain that $\mathrm{B}_{\mathrm{i}}>0, \forall \mathrm{i}=1,3$, further $\Delta>0$ if and only if:

$\mathrm{u}_{6}<\mathrm{u}_{8}\left(1+\mathrm{z}_{4}\right)$

$\mathrm{u}_{2}<\mathrm{x}_{4}$

So, according to Routh-Hurwitz criterion the roots of the third degree polynomial in the characteristic equation have negative real parts and hence the equilibrium point $\mathrm{E}_{4}$ is locally asymptotically stable.

- The Jacobian matrix of system (2) at $E_{5}$ can be written as:

$\mathrm{J}_{5}=\left[\begin{array}{cccc}-\left(1+\mathrm{y}_{5}+\mathrm{z}_{5}\right) & \mathrm{u}_{1}-\mathrm{x}_{5} & \mathrm{u}_{2}-\mathrm{x}_{5} & 1 \\ \mathrm{u}_{3} \mathrm{y}_{5} & 0 & 0 & -\mathrm{u}_{5} \mathrm{y}_{5} \\ \mathrm{u}_{6} \mathrm{z}_{5} & 0 & 0 & -\mathrm{u}_{8} \mathrm{z}_{5} \\ 0 & 0 & 0 & \mathrm{~s}_{1} \mathrm{y}_{5}-\mathrm{u}_{10} \mathrm{z}_{5}-\mathrm{u}_{11}\end{array}\right]=\left(\mathrm{c}_{\mathrm{ij}}\right)_{4 \times 4}$

The characteristic equation of $\mathrm{J}_{5}$ can be written as:

$\left(\mathrm{c}_{44}-\lambda_{5 \mathrm{w}}\right)\left(\lambda_{5}\right)\left[\lambda_{5}^{2}-\mathrm{c}_{11} \lambda_{5}-\left(\mathrm{c}_{13} \mathrm{c}_{31}+\mathrm{c}_{21} \mathrm{c}_{12}\right)\right]=0$ 
Clearly, the equilibrium point $E_{5}$ has a zero eigenvalue that's mean its non-hyperbolic point. So, the linearization failed and we will study the stability of $E_{5}$ by Lyapunov method in the next section.

- The Jacobian matrix of system (2) at $\mathrm{E}_{6}$ can be written as:

$\mathrm{J}_{6}=\left[\begin{array}{cccc}-\left(1+\mathrm{y}_{6}+\mathrm{z}_{6}\right) & \mathrm{u}_{1}-\mathrm{x}_{6} & \mathrm{u}_{2}-\mathrm{x}_{6} & 1 \\ \mathrm{u}_{3} \mathrm{y}_{6} & 0 & 0 & -\mathrm{u}_{5} \mathrm{y}_{6} \\ \mathrm{u}_{6} \mathrm{z}_{6} & 0 & 0 & -\mathrm{u}_{8} \mathrm{z}_{6} \\ 0 & \mathrm{~s}_{1} \mathrm{w}_{6} & \mathrm{u}_{10} \mathrm{w}_{6} & \mathrm{~s}_{1} \mathrm{y}_{6}-\mathrm{u}_{10} \mathrm{z}_{6}-\mathrm{u}_{11}\end{array}\right]=\left(\mathrm{d}_{\mathrm{ij}}\right)_{4 \times 4}(16 \mathrm{a})$

The characteristic equation of $\mathrm{J}_{6}$ can be written as:

$\lambda_{6}^{4}+\mathrm{D}_{1} \lambda_{6}^{3}+\mathrm{D}_{2} \lambda_{6}^{2}+\mathrm{D}_{3} \lambda_{6}+\mathrm{D}_{4}=0$

Here

$D_{1}=-\left(d_{11}+d_{44}\right)$

$D_{2}=-\left(d_{11} d_{44}+d_{12} d_{21}+d_{13} d_{31}+d_{24} d_{42}+d_{34} d_{43}\right)$

$D_{3}=d_{43}\left(d_{11} d_{34}-d_{31}\right)+d_{42}\left(d_{11} d_{24}-d_{21}\right)+d_{44}\left(d_{12} d_{21}-d_{13} d_{31}\right)$

$D_{4}=d_{12} d_{21} d_{34} d_{43}+d_{13} d_{31} d_{42} d_{24}-d_{12} d_{31} d_{24} d_{43}-d_{13} d_{21} d_{42} d_{34}$

Consequently,

$\Delta_{1}=\mathrm{D}_{1} \mathrm{D}_{2}-\mathrm{D}_{3}=\mathrm{d}_{11} \Gamma_{2}+\mathrm{d}_{44} \Gamma_{3}-\mathrm{d}_{11} \mathrm{~d}_{44} \Gamma_{1}+\Gamma_{4}$

And

$\Delta_{2}=D_{3}\left(D_{1} D_{2}-D_{3}\right)-D_{1}^{2} D_{4}=F_{1}+F_{2}+F_{3}+F_{4}$

Where

$F_{1}=\left(d_{11} \Gamma_{3}+d_{44} \Gamma_{2}\right)\left(d_{11} \Gamma_{2}+d_{44} \Gamma_{3}+\Gamma_{4}\right)$

$F_{2}=-\left(d_{11} \Gamma_{3}+d_{44} \Gamma_{2}\right)\left(d_{11} d_{44} \Gamma_{1}\right)$

$F_{3}=-\Gamma_{4}\left(\mathrm{~d}_{11} \mathrm{~d}_{44} \Gamma_{1}\right)\left(\Gamma_{2}+\mathrm{d}_{44} \Gamma_{3}+\Gamma_{4}\right)$

$\mathrm{F}_{4}=\Gamma_{1}^{2} \Gamma_{5}\left(\mathrm{~d}_{13} \mathrm{~d}_{42}-\mathrm{d}_{12} \mathrm{~d}_{43}\right)$

With

$\Gamma_{1}=d_{11}+d_{44}$

$\Gamma_{2}=\mathrm{d}_{12} \mathrm{~d}_{21}+\mathrm{d}_{13} \mathrm{~d}_{31}$

$\Gamma_{3}=\mathrm{d}_{24} \mathrm{~d}_{42}+\mathrm{d}_{34} \mathrm{~d}_{43}$

$\Gamma_{4}=\mathrm{d}_{21} \mathrm{~d}_{42}+\mathrm{d}_{31} \mathrm{~d}_{43}$

$\Gamma_{5}=\mathrm{d}_{21} \mathrm{~d}_{34}-\mathrm{d}_{31} \mathrm{~d}_{24}$

$\Gamma_{6}=\mathrm{d}_{13} \mathrm{~d}_{42}-\mathrm{d}_{12} \mathrm{~d}_{43}$

Now, according to existence condition (8c) it is observed that $D_{i}>0, i=1,3,4$, further $\Delta_{2}>0$ if and only if the following conditions hold: $\mathrm{u}_{11}>\mathrm{s}_{1} \mathrm{y}_{6}+\mathrm{u}_{10} \mathrm{z}_{6}$

$u_{2}>x_{6}>\max \left\{\frac{u_{1} u_{10}-u_{2} s_{1}}{u_{10}-s_{1}}, u_{1}\right\}$

$\mathrm{y}_{6}>\max \left\{\frac{\mathrm{u}_{6}}{\mathrm{u}_{8}}-\mathrm{z}_{6}-1, \frac{\mathrm{u}_{3}}{\mathrm{u}_{5}}-\mathrm{z}_{6}-1, \frac{\left(\mathrm{u}_{2}-\mathrm{x}_{6}\right) \mathrm{u}_{6} \mathrm{z}_{6}}{\left(\mathrm{u}_{1}-\mathrm{x}_{6}\right) \mathrm{u}_{3}}\right\}$

So, according to Routh-Hurwitz criterion the roots of the third degree polynomial in the characteristic equation have negative real parts and hence the equilibrium point $\mathrm{E}_{6}$ is locally asymptotically stable.

\section{Global stability analysis}

In In this section the global stability for the equilibrium points of system (2) is studied analytically by using the Lyapunov method as shown in the following theorems:

Theorem 2: Assume that, the equilibrium point $E_{0}$ of system (2) is locally asymptotically stable and the following conditions hold:

$\mathrm{u}_{3}<\min \left\{\frac{\mathrm{u}_{4}}{1+\mathrm{u}_{1}}, \frac{\mathrm{u}_{5} \mathrm{u}_{11}}{\mathrm{~s}_{1}}\right\}$

$\mathrm{u}_{6}<\min \left\{\frac{\mathrm{u}_{7}}{1+\mathrm{u}_{2}}, \frac{\mathrm{u}_{8} \mathrm{u}_{11}}{\mathrm{u}_{10}}\right\}$

Then $E_{0}$ is globally asymptotically stable in the $R_{+}^{4}$.

Proof: Consider the following function:

$\mathrm{V}_{0}=\mathrm{c}_{1}(\mathrm{x}-1-\ln (\mathrm{x}))+\mathrm{c}_{2} \mathrm{y}+\mathrm{c}_{3} \mathrm{z}+\mathrm{c}_{4} \mathrm{w}$

Where $c_{i}, i=1,2,3,4$ are positive constants to be determine. Clearly $V_{0}: R_{+}^{4} \rightarrow R$ is $C^{1}$ positive definite function. Now by differentiating $V_{0}$ with respect to time $t$, we get:

$$
\begin{aligned}
\frac{d v_{0}}{d t}= & -\frac{c_{1}}{x}(x-1)^{2}+\left(c_{1}\left(1+u_{1}\right)-c_{2} u_{4}\right) y+\left(c_{1}\left(1+u_{2}\right)-c_{3} u_{7}\right) z \\
& +\left(c_{1}-c_{4} u_{11}\right) w+\left(c_{2} u_{3}-c_{1}\right) x y+\left(c_{3} u_{6}-c_{1}\right) x z \\
& +\left(c_{4} s_{1}-c_{2} u_{5}\right) y w+\left(c_{4} u_{10}-c_{3} u_{8}\right) z w-\frac{c_{1}}{x}\left(u_{1} y+u_{2} z+w\right)
\end{aligned}
$$

So by choosing the positive constants as below:

$\mathrm{c}_{1}=1, \mathrm{c}_{2}=\frac{1}{\mathrm{u}_{3}}, \mathrm{c}_{3}=\frac{1}{\mathrm{u}_{6}}, \mathrm{c}_{4}=\frac{1}{\mathrm{u}_{11}}$

We obtain that:

$$
\begin{aligned}
\frac{\mathrm{dv}_{0}}{\mathrm{dt}} \leq & -\frac{(\mathrm{x}-1)^{2}}{\mathrm{x}}+\left(1+\mathrm{u}_{1}-\frac{\mathrm{u}_{4}}{\mathrm{u}_{3}}\right) \mathrm{y}+\left(1+\mathrm{u}_{2}-\frac{\mathrm{u}_{7}}{\mathrm{u}_{6}}\right) \mathrm{z} \\
& +\left(\frac{\mathrm{s}_{1}}{\mathrm{u}_{11}}-\frac{\mathrm{u}_{5}}{\mathrm{u}_{3}}\right) \mathrm{yw}+\left(\frac{\mathrm{u}_{10}}{\mathrm{u}_{11}}-\frac{\mathrm{u}_{8}}{\mathrm{u}_{6}}\right) \mathrm{zw}
\end{aligned}
$$


According to conditions (17)(a,b) we have $\frac{\mathrm{dV}_{0}}{\mathrm{dt}}<0$. Therefore $\mathrm{E}_{0}$ is globally asymptotically stable in the $\mathrm{R}_{+}^{4}$, and hence the proof is complete.

Theorem 3: Assume that, the equilibrium point $E_{1}$ of system (2) is locally asymptotically stable. Then the basin of attraction of $E_{1}$, say $B\left(E_{1}\right) \subset R_{+}^{4}$, satisfy the following conditions:

$$
\left(u_{1}-x_{1}+u_{3} y\right)^{2} \leq 4(1+y)\left(u_{4}-u_{3} x_{1}\right)
$$

$\mathrm{x}_{1}+\mathrm{u}_{2}+\frac{\mathrm{u}_{6}}{\mathrm{u}_{8}}<\mathrm{x}<\mathrm{x}_{1}+\frac{\mathrm{u}_{11}}{\mathrm{u}_{10}}$

$\mathrm{y}>\mathrm{y}_{1}+\frac{\mathrm{s}_{1}}{\mathrm{u}_{5} \mathrm{u}_{10}}$

Proof: Consider the following function:

$\mathrm{v}_{1}=\mathrm{c}_{1} \frac{\left(\mathrm{x}-\mathrm{x}_{1}\right)^{2}}{2}+\mathrm{c}_{2} \frac{\left(\mathrm{y}-\mathrm{y}_{1}\right)^{2}}{2}+\mathrm{c}_{3} \mathrm{z}+\mathrm{c}_{4} \mathrm{w}$

Where $c_{i}, i=1,2,3,4$ are positive constant to be determine. Clearly $V_{1}: R_{+}^{4} \rightarrow R$ is $C^{1}$ positive definite function. Now by differentiating $V_{1}$ with respect to time $t$, we get:

$$
\begin{aligned}
& \frac{\mathrm{dV}_{1}}{\mathrm{dt}}=-\mathrm{c}_{1}(1+\mathrm{y})\left(\mathrm{x}-\mathrm{x}_{1}\right)^{2}+\left(\mathrm{c}_{1}\left(\mathrm{u}_{1}-\mathrm{x}_{1}\right)+\mathrm{c}_{2} \mathrm{u}_{3} \mathrm{y}\right)\left(\mathrm{x}-\mathrm{x}_{1}\right)\left(\mathrm{y}-\mathrm{y}_{1}\right) \\
& -\mathrm{c}_{2}\left(\mathrm{u}_{4}-\mathrm{u}_{3} \mathrm{x}_{1}\right)\left(\mathrm{y}-\mathrm{y}_{1}\right)^{2}-\left(\mathrm{c}_{1} \mathrm{u}_{2} \mathrm{x}_{1}+\mathrm{c}_{3} \mathrm{u}_{7}\right) \mathrm{z} \\
& +\left(\mathrm{c}_{1}\left(\mathrm{x}-\mathrm{x}_{1}\right)-\mathrm{c}_{4} \mathrm{u}_{11}\right) \mathrm{w}+\left(\mathrm{c}_{1}\left(\mathrm{x}_{1}+\mathrm{u}_{2}-\mathrm{x}\right)+\mathrm{c}_{3} \mathrm{u}_{6}\right) \mathrm{xz} \\
& +\left(\mathrm{c}_{4} \mathrm{~s}_{1}-\mathrm{c}_{2} \mathrm{u}_{5}\left(\mathrm{y}-\mathrm{y}_{1}\right)\right) \mathrm{yw}+\left(\mathrm{c}_{4} \mathrm{u}_{10}-\mathrm{c}_{3} \mathrm{u}_{8}\right) \mathrm{zw}
\end{aligned}
$$

So by choosing the positive constants as below:

$$
\mathrm{c}_{1}=1, \mathrm{c}_{2}=1, \mathrm{c}_{3}=\frac{1}{\mathrm{u}_{8}}, \mathrm{c}_{4}=\frac{1}{\mathrm{u}_{10}}
$$

And according to condition (18a) we obtain that:

$$
\begin{aligned}
& \frac{\mathrm{dV}_{1}}{\mathrm{dt}} \leq-\left[\sqrt{(1+\mathrm{y})}\left(\mathrm{x}-\mathrm{x}_{1}\right)-\sqrt{\left(\mathrm{u}_{4}-\mathrm{u}_{3} \mathrm{x}_{1}\right)}\left(\mathrm{y}-\mathrm{y}_{1}\right)\right]^{2} \\
& +\left(\mathrm{x}-\mathrm{x}_{1}-\frac{\mathrm{u}_{11}}{\mathrm{u}_{10}}\right) \mathrm{w}+\left(\mathrm{x}_{1}+\mathrm{u}_{2}-\mathrm{x}+\frac{\mathrm{u}_{6}}{\mathrm{u}_{8}}\right) \mathrm{xz} \\
& +\left(\frac{\mathrm{s}_{1}}{\mathrm{u}_{10}}-\mathrm{u}_{5}\left(\mathrm{y}-\mathrm{y}_{1}\right)\right) \mathrm{yw}
\end{aligned}
$$

Obviously $\frac{\mathrm{dV}_{1}}{\mathrm{dt}}<0$ for every initial point satisfying conditions $(18)(b, c)$ and then $V_{1}$ is a Lyapunov function provided that conditions (18)(a-c) hold. Thus $E_{1}$ is globally asymptotically stable in the interior of $\mathrm{B}\left(\mathrm{E}_{1}\right)$, which means that $\mathrm{B}\left(\mathrm{E}_{1}\right)$ is the basin of attraction and this completes the proof.

Theorem 4: Assume that, the equilibrium point $E_{2}$ of system (2) is locally asymptotically stable. Then the basin of attraction of $E_{2}$, say $B\left(E_{2}\right) \subset R_{+}^{4}$, satisfy the following conditions: $\left(u_{2}-x_{2}+u_{6} z\right)^{2} \leq 4(1+z)\left(u_{7}-u_{6} x_{2}\right)$

$\mathrm{x}_{2}+\mathrm{u}_{1}+\frac{\mathrm{u}_{3}}{\mathrm{u}_{5}}<\mathrm{x}<\mathrm{x}_{2}+\frac{\mathrm{u}_{11}}{\mathrm{~s}_{1}}$

$$
\mathrm{z}>\mathrm{z}_{2}+\frac{\mathrm{u}_{10}}{\mathrm{~s}_{1} \mathrm{u}_{8}}
$$

Proof: Consider the following function:

$\mathrm{v}_{2}=\mathrm{c}_{1} \frac{\left(\mathrm{x}-\mathrm{x}_{2}\right)^{2}}{2}+\mathrm{c}_{2} \mathrm{y}+\mathrm{c}_{3} \frac{\left(\mathrm{z}-\mathrm{z}_{2}\right)^{2}}{2}+\mathrm{c}_{4} \mathrm{w}$

Where $c_{i}, i=1,2,3,4$ are positive constants to be determine. Clearly $V_{2}: R_{+}^{4} \rightarrow R$ is $C^{1}$ positive definite function. Now by differentiating $V_{2}$ with respect to time $t$, we get:

$$
\begin{aligned}
\frac{\mathrm{dV}_{2}}{\mathrm{dt}}= & -\mathrm{c}_{1}(1+\mathrm{z})\left(\mathrm{x}-\mathrm{x}_{2}\right)^{2}+\left(\mathrm{c}_{1}\left(\mathrm{u}_{2}-\mathrm{x}_{2}\right)+\mathrm{c}_{3} \mathrm{u}_{6} \mathrm{z}\right)\left(\mathrm{x}-\mathrm{x}_{2}\right)\left(\mathrm{z}-\mathrm{z}_{2}\right) \\
& -\mathrm{c}_{3}\left(\mathrm{u}_{7}-\mathrm{u}_{6} \mathrm{x}_{2}\right)\left(\mathrm{z}-\mathrm{z}_{2}\right)^{2}-\left(\mathrm{c}_{1} \mathrm{u}_{1} \mathrm{x}_{2}+\mathrm{c}_{2} \mathrm{u}_{4}\right) \mathrm{y} \\
& +\left(\mathrm{c}_{1}\left(\mathrm{x}_{-}-\mathrm{x}_{2}\right)-\mathrm{c}_{4} \mathrm{u}_{11}\right) \mathrm{w}+\left(\mathrm{c}_{1}\left(\mathrm{x}_{2}+\mathrm{u}_{1}-\mathrm{x}\right)+\mathrm{c}_{2} \mathrm{u}_{3}\right) \mathrm{xy} \\
& +\left(c_{4} s_{1}-c_{2} u_{5}\right) y w+\left(c_{4} u_{10}-c_{3} u_{8}\left(z-z_{2}\right)\right) z w
\end{aligned}
$$

So by choosing the positive constants as below:

$\mathrm{c}_{1}=1, \mathrm{c}_{2}=\frac{1}{\mathrm{u}_{5}}, \mathrm{c}_{3}=1, \mathrm{c}_{4}=\frac{1}{\mathrm{~s}_{1}}$

And according to condition (19a) we obtain that:

$$
\begin{aligned}
\frac{\mathrm{dV}_{2}}{\mathrm{dt}} \leq & -\left[\sqrt{(1+\mathrm{z})}\left(\mathrm{x}-\mathrm{x}_{2}\right)-\sqrt{\left(\mathrm{u}_{7}-\mathrm{u}_{6} \mathrm{x}_{2}\right)}\left(\mathrm{z}-\mathrm{z}_{2}\right)\right]^{2} \\
& +\left(\mathrm{x}-\mathrm{x}_{2}-\frac{\mathrm{u}_{11}}{\mathrm{~s}_{1}}\right) \mathrm{w}+\left(\mathrm{x}_{2}+\mathrm{u}_{1}-\mathrm{x}+\frac{\mathrm{u}_{3}}{\mathrm{u}_{5}}\right) \mathrm{xy} \\
& +\left(\frac{\mathrm{u}_{10}}{\mathrm{~s}_{1}}-\mathrm{u}_{8}\left(\mathrm{z}-\mathrm{z}_{2}\right)\right) \mathrm{zw}
\end{aligned}
$$

Obviously $\frac{\mathrm{dV}_{2}}{\mathrm{dt}}<0$ for every initial point satisfying conditions (19)(b,c) and then $v_{2}$ is a Lyapunov function provided that conditions (19)(a-c) hold. Thus $E_{2}$ is globally asymptotically stable in the interior of $\mathrm{B}\left(\mathrm{E}_{2}\right)$, which means that $\mathrm{B}\left(\mathrm{E}_{2}\right)$ is the basin of attraction and this completes the proof.

Theorem 5: Assume that, the equilibrium point $E_{3}$ of system (2) is locally asymptotically stable. Then the basin of attraction of $E_{3}$, say $B\left(E_{3}\right) \subset R_{+}^{4}$, satisfy the following conditions:

$$
\begin{aligned}
& \left(u_{1}-x_{3}+u_{3} y\right)^{2} \leq(1+y)\left(u_{4}+u_{5} w-u_{3} x_{3}\right) \\
& \left(s_{1} w-u_{5} y_{3}\right)^{2} \leq\left(u_{4}+u_{5} w-u_{3} x_{3}\right)\left(u_{11}-s_{1} y_{3}\right) \\
& (1+y)\left(u_{11}-s_{1} y_{3}\right) \geq 1 \\
& x>x_{3}+u_{2}+u_{6}
\end{aligned}
$$


$\mathrm{w}<\mathrm{w}_{3}$

$(20 \mathrm{e})$

Proof: Consider the following function:

$$
\mathrm{V}_{3}=\frac{\left(\mathrm{x}-\mathrm{x}_{3}\right)^{2}}{2}+\frac{\left(\mathrm{y}-\mathrm{y}_{3}\right)^{2}}{2}+\mathrm{z}+\frac{\left(\mathrm{w}-\mathrm{w}_{3}\right)^{2}}{2}
$$

Clearly $V_{3}: R_{+}^{4} \rightarrow R$ is $C^{1}$ positive definite function. Now by differentiating $V_{3}$ with respect to time $t$, and according to conditions (20)(a-c) we obtain that:

$$
\begin{aligned}
\frac{\mathrm{dV}_{3}}{\mathrm{dt} \leq} & -\left[\sqrt{\frac{(1+\mathrm{y})}{2}}\left(\mathrm{x}-\mathrm{x}_{3}\right)-\sqrt{\frac{\left(\mathrm{u}_{4}+\mathrm{u}_{5} \mathrm{w}-\mathrm{u}_{3} \mathrm{x}_{3}\right)}{2}}\left(y-\mathrm{y}_{3}\right)\right]^{2} \\
& -\left[\sqrt{\frac{(1+\mathrm{y})}{2}}\left(\mathrm{x}-\mathrm{x}_{3}\right)-\sqrt{\frac{\left(\mathrm{u}_{11}-\mathrm{s}_{1} \mathrm{y}_{3}\right)}{2}}\left(\mathrm{w}-\mathrm{w}_{3}\right)\right]^{2} \\
& -\left[\sqrt{\frac{\left(u_{4}+\mathrm{u}_{5} \mathrm{w}-\mathrm{u}_{3} \mathrm{x}_{3}\right)}{2}}\left(\mathrm{y}-\mathrm{y}_{3}\right)-\sqrt{\frac{\left(u_{11}-\mathrm{s}_{1} \mathrm{y}_{3}\right)}{2}}\left(w-w_{3}\right)\right]^{2} \\
& +\left(\mathrm{x}_{3}+\mathrm{u}_{2}-\mathrm{x}+\mathrm{u}_{6}\right) \mathrm{xz}^{2}+\mathrm{u}_{10}\left(\mathrm{w}-\mathrm{w}_{3}\right) \mathrm{zw}
\end{aligned}
$$

Obviously $\frac{\mathrm{dV}_{3}}{\mathrm{dt}}<0$, and then $\mathrm{V}_{3}$ is a Lyapunov function provided that the given conditions hold. Therefore $\mathrm{E}_{3}$ is globally asymptotically stable in the interior of $B\left(E_{3}\right)$, which means that $B\left(E_{3}\right)$ is the basin of attraction of $E_{3}$ and the proof is complete.

Theorem 6: Assume that, the equilibrium point $E_{4}$ of system (2) is locally asymptotically stable. Then the basin of attraction of $E_{4}$, say $B\left(E_{4}\right) \subset R_{+}^{4}$, satisfy the following conditions:

$$
\begin{aligned}
& \left(u_{2}-x_{4}+u_{6} z\right)^{2} \leq(1+z)\left(u_{7}+u_{8} w-u_{6} x_{4}\right) \\
& \left(u_{10} w-u_{8} z_{4}\right)^{2} \leq\left(u_{7}+u_{8} w-u_{6} x_{4}\right)\left(u_{11}-u_{10} z_{4}\right) \\
& (1+z)\left(u_{11}-u_{10} z_{4}\right) \geq 1 \\
& x>x_{4}+u_{1}+u_{3} \\
& w<w_{4}
\end{aligned}
$$

Proof: Consider the following function:

$$
\mathrm{v}_{4}=\frac{\left(\mathrm{x}-\mathrm{x}_{4}\right)^{2}}{2}+\mathrm{y}+\frac{\left(\mathrm{z}-\mathrm{z}_{4}\right)^{2}}{2}+\frac{\left(\mathrm{w}-\mathrm{w}_{4}\right)^{2}}{2}
$$

Clearly $\mathrm{V}_{4}: \mathrm{R}_{+}^{4} \rightarrow \mathrm{R}$ is $\mathrm{C}^{1}$ positive definite function. Now by differentiating $\mathrm{V}_{4}$ with respect to time $t$, and according to conditions (21)(a-c) we obtain that:

$$
\begin{aligned}
\frac{\mathrm{dV}_{4}}{\mathrm{dt}} \leq & -\left[\sqrt{\frac{(1+\mathrm{z})}{2}}\left(\mathrm{x}-\mathrm{x}_{4}\right)-\sqrt{\frac{\left(\mathrm{u}_{7}+\mathrm{u}_{8} \mathrm{w}-\mathrm{u}_{6} \mathrm{x}_{4}\right)}{2}}\left(\mathrm{z}-\mathrm{z}_{4}\right)\right]^{2} \\
& -\left[\sqrt{\frac{(1+\mathrm{z})}{2}}\left(\mathrm{x}-\mathrm{x}_{4}\right)-\sqrt{\frac{\left(\mathrm{u}_{11}-\mathrm{u}_{\left.10 \mathrm{z}_{4}\right)}\right.}{2}}\left(\mathrm{w}-\mathrm{w}_{4}\right)\right]^{2}
\end{aligned}
$$

$$
\begin{aligned}
& -\left[\sqrt{\frac{\left(u_{7}+u_{8} w-u_{6} x_{4}\right)}{2}}\left(z-z_{4}\right)-\sqrt{\frac{\left(u_{11}-u_{10} z_{4}\right)}{2}}\left(w-w_{4}\right)\right]^{2} \\
& +\left(x_{4}+u_{1}-x+u_{3}\right) x_{1}+s_{1}\left(w-w_{4}\right) y w
\end{aligned}
$$

Obviously $\frac{\mathrm{dV}_{4}}{\mathrm{dt}}<0$, and then $\mathrm{V}_{4}$ is a Lyapunov function provided that the given conditions hold. Therefore $\mathrm{E}_{4}$ is globally asymptotically stable in the interior of $\mathrm{B}\left(\mathrm{E}_{4}\right)$, which means that $\mathrm{B}\left(\mathrm{E}_{4}\right)$ is the basin of attraction of $\mathrm{E}_{4}$ and the proof is complete.

Theorem 7: Assume that, the equilibrium point $E_{5}$ of system (2) is locally asymptotically stable. Then the basin of attraction of $E_{5}$, say $B\left(E_{5}\right) \subset R_{+}^{4}$, satisfy the following conditions:

$$
\begin{aligned}
& \left(u_{1}-x_{5}+u_{3} y\right)^{2} \leq 2(1+y+z)\left(u_{4}-u_{3} x_{5}\right) \\
& \left(u_{2}-x_{5}+u_{6} z\right)^{2} \leq 2(1+y+z)\left(u_{7}-u_{6} x_{5}\right) \\
& u_{11}>x+\left(u_{5} y_{5}+s_{1}\right) y+\left(u_{8} z_{5}+u_{10}\right) z
\end{aligned}
$$

Proof: Consider the following function:

$$
\mathrm{V}_{5}=\frac{\left(\mathrm{x}-\mathrm{x}_{5}\right)^{2}}{2}+\frac{\left(\mathrm{y}-\mathrm{y}_{5}\right)^{2}}{2}+\frac{\left(\mathrm{z}-\mathrm{z}_{5}\right)^{2}}{2}+\mathrm{w}
$$

Clearly $V_{5}: R_{+}^{4} \rightarrow R$ is $C^{1}$ positive definite function. Now by differentiating $\mathrm{V}_{5}$ with respect to time $t$, and according to conditions (22)(a,b) we obtain that:

$$
\begin{aligned}
\frac{\mathrm{dV}_{5}}{\mathrm{dt}} \leq & -\left[\sqrt{\frac{(1+\mathrm{y}+\mathrm{z})}{2}}\left(x-x_{5}\right)-\sqrt{\left(u_{4}-\mathrm{u}_{3} \mathrm{x}_{5}\right)}\left(y-y_{5}\right)\right]^{2} \\
& -\left[\sqrt{\frac{(1+\mathrm{y}+\mathrm{z})}{2}}\left(\mathrm{x}-\mathrm{x}_{5}\right)-\sqrt{\left(\mathrm{u}_{7}-\mathrm{u}_{6} \mathrm{z}_{5}\right)}\left(\mathrm{z}-\mathrm{z}_{5}\right)\right]^{2} \\
& +\left[\mathrm{x}-\mathrm{u}_{11}+\left(\mathrm{s}_{1}+\mathrm{y}_{5}\right) \mathrm{y}+\left(\mathrm{u}_{10}-\mathrm{z}_{5}\right) \mathrm{z}\right] \mathrm{w}
\end{aligned}
$$

Obviously $\frac{\mathrm{dV}_{5}}{\mathrm{dt}}<0$, and then $\mathrm{V}_{5}$ is a Lyapunov function provided that the given conditions hold. Therefore $E_{5}$ is globally asymptotically stable in the interior of $B\left(E_{5}\right)$, which means that $B\left(E_{5}\right)$ is the basin of attraction of $E_{5}$ and the proof is complete.

Theorem 8: Assume that, the equilibrium point $E_{6}$ of system (2) is locally asymptotically stable. Then the basin of attraction of $E_{6}$, say $B\left(E_{6}\right) \subset R_{+}^{4}$, satisfy the following conditions:

$$
\left(u_{1}-x_{6}+u_{3} y\right)^{2} \leq \frac{2}{3}(1+y+z)\left(u_{4}-u_{3} x_{6}+u_{5} w\right)
$$

$$
\left(u_{2}-x_{6}+u_{6}\right)^{2} \leq \frac{2}{3}(1+y+z)\left(u_{7}-u_{6} x_{6}+u_{8} w\right)
$$

$\frac{4}{9}(1+\mathrm{y}+\mathrm{z})\left(\mathrm{u}_{11}-\mathrm{s}_{1} \mathrm{y}_{6}-\mathrm{u}_{10} \mathrm{z}_{4}\right) \geq 1$

$$
\left(\mathrm{s}_{1} \mathrm{w}-\mathrm{u}_{5} \mathrm{y}_{6}\right)^{2} \leq \frac{2}{3}\left(\mathrm{u}_{4}-\mathrm{u}_{3} \mathrm{x}_{6}+\mathrm{u}_{5} \mathrm{w}\right)\left(\mathrm{u}_{11}-\mathrm{s}_{1} \mathrm{y}_{6}-\mathrm{u}_{10} \mathrm{z}\right)
$$


$\left(u_{10} w-u_{8} z_{6}\right)^{2} \leq \frac{2}{3}\left(u_{7}-u_{6} x_{6}+u_{8} w\right)\left(u_{11}-s_{1} y_{6}-u_{10} z\right)$

Proof: Consider the following function:

$\mathrm{V}_{6}=\frac{\left(\mathrm{x}-\mathrm{x}_{6}\right)^{2}}{2}+\frac{\left(\mathrm{y}-\mathrm{y}_{6}\right)^{2}}{2}+\frac{\left(\mathrm{z}-\mathrm{z}_{6}\right)^{2}}{2}+\frac{\left(\mathrm{w}-\mathrm{w}_{6}\right)^{2}}{2}$

Clearly $\mathrm{V}_{6}: \mathrm{R}_{+}^{4} \rightarrow \mathrm{R}$ is $\mathrm{C}^{1}$ positive definite function. Now by differentiating $V_{6}$ with respect to time $t$, and according to conditions (23)(a-e) we obtain that:

$$
\begin{aligned}
& \frac{\mathrm{dV}_{6}}{\mathrm{dt}} \leq-\left[\sqrt{\frac{(1+\mathrm{y}+\mathrm{z})}{3}}\left(\mathrm{x}-\mathrm{x}_{6}\right)-\sqrt{\frac{\left(\mathrm{u}_{4}-\mathrm{u}_{3} \mathrm{x}_{6}+\mathrm{u}_{5} \mathrm{w}\right)}{2}}\left(\mathrm{y}-\mathrm{y}_{6}\right)\right]^{2} \\
& -\left[\sqrt{\frac{(1+\mathrm{y}+\mathrm{z})}{3}}\left(\mathrm{x}-\mathrm{x}_{6}\right)-\sqrt{\frac{\left(\mathrm{u}_{7}-\mathrm{u}_{6} \mathrm{x}_{6}+\mathrm{u}_{8} \mathrm{w}\right)}{2}}\left(\mathrm{z}-\mathrm{z}_{6}\right)\right]^{2} \\
& -\left[\sqrt{\frac{(1+\mathrm{y}+\mathrm{z})}{3}}\left(\mathrm{x}-\mathrm{x}_{6}\right)-\sqrt{\frac{\left(\mathrm{u}_{11}-\mathrm{s}_{1} \mathrm{y}_{6}-\mathrm{u}_{10} \mathrm{z}\right)}{3}}\left(\mathrm{w}-\mathrm{w}_{6}\right)\right]^{2} \\
& -\left[\sqrt{\frac{\left(\mathrm{u}_{4}-\mathrm{u}_{3} \mathrm{x}_{6}+\mathrm{u}_{5} \mathrm{w}\right)}{2}}\left(\mathrm{y}-\mathrm{y}_{6}\right)-\sqrt{\frac{\left(\mathrm{u}_{11}-\mathrm{s}_{1} \mathrm{y}_{6}-\mathrm{u}_{10} \mathrm{z}\right)}{3}}\left(\mathrm{w}-\mathrm{w}_{6}\right)\right]^{2} \\
& -\left[\sqrt{\frac{\left(u_{7}-u_{6} x_{6}+u_{8} w\right)}{2}}\left(z-z_{6}\right)-\sqrt{\frac{\left(u_{11}-s_{1} y_{6}-u_{10} z\right)}{3}}\left(w-w_{6}\right)\right]^{2}
\end{aligned}
$$

Obviously $\frac{\mathrm{dV}_{6}}{\mathrm{dt}}<0$, and then $\mathrm{V}_{6}$ is a Lyapunov function provided that the given conditions hold. Therefore $\mathrm{E}_{6}$ is globally asymptotically stable in the interior of $B\left(E_{6}\right)$, which means that $B\left(E_{6}\right)$ is the basin of attraction of $\mathrm{E}_{6}$ and the proof is complete.

\section{The local bifurcation analysis}

In this section, the local bifurcation near the equilibrium points of the system (2) is investigated by using the Sotomayor's theorem [14] for local bifurcation. It is well known that the existence of nonhyperbolic equilibrium point is a necessary but not sufficient condition for bifurcation to occur. Now, according to Jacobian matrix of system (2) given in equation (9), it is clear to verify that for any non-zero vector $\mathrm{V}=\left(\mathrm{v}_{1}, \mathrm{v}_{2}, \mathrm{v}_{3}, \mathrm{v}_{4}\right)^{\mathrm{T}}$ we have:

$$
D^{2} F(V, V)=\left(\begin{array}{c}
-2 v_{1}\left(v_{2}+v_{3}\right) \\
2 v_{2}\left(u_{3} v_{1}-u_{5} v_{4}\right) \\
2 v_{3}\left(u_{6} v_{1}-u_{8} v_{4}\right) \\
2 v_{4}\left(s_{1} v_{2}+u_{10} v_{3}\right)
\end{array}\right)
$$

Here $\mathrm{D}^{2}$ represent the derivative of Jacobian matrix of system (2), and $\mathrm{F}=\left(\mathrm{F}_{1}, \mathrm{~F}_{2}, \mathrm{~F}_{3}, \mathrm{~F}_{4}\right)^{\mathrm{T}}$ with $\mathrm{F}_{\mathrm{i}}, \mathrm{i}=1,2,3,4$ given in system (2). Therefore, $D^{3} F(V, V, V)=(0,0,0,0)^{\mathrm{T}}$.

So, according to Sotomayor's theorem the pitchfork bifurcation does not occur at each point $E_{i}, i=0,1,2,3,4,5,6$.

Theorem 9: Assume that the local stability condition (10b) holds, and let the parameter value $u_{6}$ passing through the value $u_{6}^{*}=u_{7}$ then the system (2) at the equilibrium point $E_{0}$ has:
1) No saddle-node bifurcation.

2) Transcritical bifurcation.

Proof: According to the Jacobian matrix $\mathrm{J}_{0}$ given by Eq. (10a) the system (2) at the equilibrium point $E_{0}$ has zero eigenvalue (say $\lambda_{0 \mathrm{z}}=0$ ) at $\mathrm{u}_{6}=\mathrm{u}_{6}^{*}$, and the Jacobian matrix $\mathrm{J}_{0}$ with $\mathrm{u}_{6}=\mathrm{u}_{6}^{*}$ becomes:

$\mathrm{J}_{0}^{*}=\mathrm{J}\left(\mathrm{u}_{6}=\mathrm{u}_{6}^{*}\right)=\left(\begin{array}{cccc}-1 & \mathrm{u}_{1}-1 & \mathrm{u}_{2}-1 & 1 \\ 0 & \mathrm{u}_{3}-\mathrm{u}_{4} & 0 & 0 \\ 0 & 0 & 0 & 0 \\ 0 & 0 & 0 & -\mathrm{u}_{11}\end{array}\right)$

Now, let $\mathrm{V}^{[0]}=\left(\mathrm{v}_{1}^{[0]}, \mathrm{v}_{2}^{[0]}, \mathrm{v}_{3}^{[0]}, \mathrm{v}_{4}^{[0]}\right)^{\mathrm{T}}$ be the eigenvector corresponding to the eigenvalue $\lambda_{0 \mathrm{z}}=0$. Thus $\mathrm{J}_{0}^{*} \mathrm{~V}^{[0]}=0$, gives $\mathrm{V}^{[0]}=\left(\left(\mathrm{u}_{2}-1\right) \mathrm{v}_{3}^{[0]}, 0, \mathrm{v}_{3}^{[0]}, 0\right)^{\mathrm{T}}$, where $\mathrm{v}_{3}^{[0]}$ any nonzero real number. Let $\Psi^{[0]}=\left(\psi_{1}^{[0]}, \psi_{2}^{[0]}, \psi_{3}^{[0]}, \psi_{4}^{[0]}\right)^{\mathrm{T}}$ be the eigenvector corresponding to the eigenvalue $\lambda_{0 \mathrm{z}}=0$ of the matrix $\mathrm{J}_{0}^{* \mathrm{~T}}$.

Then $\mathrm{J}_{0}^{* \mathrm{~T}} \Psi^{[0]}=0$, by solving this equation for $\Psi^{[0]}$ we get $\Psi^{[0]}=\left(0,0, \Psi_{3}^{[0]}, 0\right)^{\mathrm{T}}$, where $\psi_{3}^{[0]}$ any nonzero real number. Now, consider:

$\frac{\partial \mathrm{f}}{\partial \mathrm{u}_{6}}=\mathrm{f}_{\mathrm{u}_{6}}\left(\mathrm{X}, \mathrm{u}_{6}\right)=\left(\frac{\partial \mathrm{f}_{1}}{\partial \mathrm{u}_{6}}, \frac{\partial \mathrm{f}_{2}}{\partial \mathrm{u}_{6}}, \frac{\partial \mathrm{f}_{3}}{\partial \mathrm{u}_{6}}, \frac{\partial \mathrm{f}_{4}}{\partial \mathrm{u}_{6}}\right)^{\mathrm{T}}=(0,0, x \mathrm{x}, 0)^{\mathrm{T}}$

Thus, $\mathrm{f}_{\mathrm{u}_{6}}\left(\mathrm{E}_{0}, \mathrm{u}_{6}^{*}\right)=(0,0,0,0)^{\mathrm{T}}$ and hence $\left(\Psi^{[0]}\right)^{\mathrm{T}} \mathrm{f}_{\mathrm{u}_{6}}\left(\mathrm{E}_{0}, \mathrm{u}_{6}^{*}\right)=0$. So, according to Sotomayor's theorem the saddle-node bifurcation cannot occur, while the first condition of transcritical bifurcation is satisfied. Now, since

$\mathrm{Df}_{\mathrm{u}_{6}}\left(X, \mathrm{u}_{6}\right)=\left(\begin{array}{cccc}0 & 0 & 0 & 0 \\ 0 & 0 & 0 & 0 \\ \mathrm{z} & 0 & \mathrm{x} & 0 \\ 0 & 0 & 0 & 0\end{array}\right)$

Where $\mathrm{Df}_{\mathrm{u}_{6}}\left(\mathrm{X}, \mathrm{u}_{6}\right)$ represents the derivative of $\mathrm{f}_{\mathrm{u}_{6}}\left(\mathrm{X}, \mathrm{u}_{6}\right)$ with respect to $\mathrm{X}=(\mathrm{x}, \mathrm{y}, \mathrm{z}, \mathrm{w})^{\mathrm{T}}$. Further, it is observed

$\mathrm{Df}_{\mathrm{u}_{6}}\left(\mathrm{E}_{0}, \mathrm{u}_{6}^{*}\right) \mathrm{V}^{[0]}=\left(\begin{array}{cccc}0 & 0 & 0 & 0 \\ 0 & 0 & 0 & 0 \\ 0 & 0 & 1 & 0 \\ 0 & 0 & 0 & 0\end{array}\right)\left(\begin{array}{c}\left(\mathrm{u}_{2}-1\right) \mathrm{v}_{3}^{[0]} \\ 0 \\ \mathrm{v}_{3}^{[0]} \\ 0\end{array}\right)=\left(\begin{array}{c}0 \\ 0 \\ \mathrm{v}_{3}^{[0]} \\ 0\end{array}\right)$

$\left(\Psi^{[0]}\right)^{\mathrm{T}}\left[\mathrm{Df}_{\mathrm{u}_{6}}\left(\mathrm{E}_{0}, \mathrm{u}_{6}^{*}\right) \mathrm{V}^{[0]}\right]=\left(0,0, \psi_{3}^{[0]}, 0\right)\left(0,0, \mathrm{v}_{3}^{[0]}, 0\right)^{\mathrm{T}}$

$$
=\mathrm{v}_{3}^{[0]} \psi_{3}^{[0]} \neq 0
$$

Now, by substituting $\mathrm{V}^{[0]}$ in (24) we get

$D^{2} f\left(E_{0}, u_{6}^{*}\right)\left(V^{[0]}, v^{[0]}\right)=\left(0,0,2 u_{6}^{*}\left(u_{2}-1\right) v_{3}^{[0]^{2}}, 0\right)^{T}$

Hence, it is obtain that:

$\left(\Psi^{[0]}\right)^{\mathrm{T}}\left[\mathrm{D}^{2} \mathrm{f}\left(\mathrm{E}_{0}, \mathrm{u}_{6}^{*}\right)\left(\mathrm{V}^{[0]}, \mathrm{V}^{[0]}\right)\right]$

$$
\begin{aligned}
& =\left(0,0, \psi_{3}^{[0]}, 0\right)\left(0,0,2 u_{6}^{*}\left(u_{2}-1\right) v_{3}^{[0]^{2}}, 0\right)^{T} \\
& =2 u_{6}^{*}\left(u_{2}-1\right) v_{3}^{[0]^{2}} \psi_{3}^{[0]} \neq 0
\end{aligned}
$$


Since $u_{2}$ represent a consumption rate then $u_{2}-1 \neq 0$. Thus, according to Sotomayor's theorem system (2) has transcritical bifurcation at $\mathrm{E}_{0}$ with the parameter $\mathrm{u}_{6}=\mathrm{u}_{6}^{*}$.

Theorem 10: Assume that the local stability condition (11c) hold and suppose that the condition

$\mathrm{u}_{1} \mathrm{u}_{5}+\mathrm{u}_{4} \neq \mathrm{u}_{1} \mathrm{u}_{3}+\mathrm{u}_{5}(25)$

Is satisfied. Then when the parameter value $u_{11}$ passing through $u_{11}^{*}=\frac{\mathrm{s}_{1}\left(\mathrm{u}_{4}-\mathrm{u}_{3}\right)}{\mathrm{u}_{1} \mathrm{u}_{3}-\mathrm{u}_{4}}$ system (2) at the equilibrium point $E_{1}$ has:

1) No saddle-node bifurcation.

2) Transcritical bifurcation.

Proof: According to the Jacobian matrix $J_{1}$ given by Eq. (11a) the system (2) at the equilibrium point $E_{1}$ has zero eigenvalue (say $\lambda_{1 \mathrm{w}}=0$ ) at $\mathrm{u}_{11}=\mathrm{u}_{11}^{*}$, and the Jacobian matrix $\mathrm{J}_{1}$ with $\mathrm{u}_{11}=\mathrm{u}_{11}^{*}$ becomes:

$$
\mathrm{J}_{1}^{*}=\mathrm{J}\left(\mathrm{u}_{11}=\mathrm{u}_{11}^{*}\right)
$$

$$
=\left(\begin{array}{cccc}
\frac{\mathrm{u}_{3}-\mathrm{u}_{1} \mathrm{u}_{3}}{\mathrm{u}_{1} \mathrm{u}_{3}-\mathrm{u}_{4}} & \frac{\mathrm{u}_{1} \mathrm{u}_{3}-\mathrm{u}_{4}}{\mathrm{u}_{3}} & \frac{\mathrm{u}_{2} \mathrm{u}_{3}-\mathrm{u}_{4}}{\mathrm{u}_{3}} & 1 \\
\frac{\mathrm{u}_{3}\left(\mathrm{u}_{4}-\mathrm{u}_{3}\right)}{\mathrm{u}_{1} \mathrm{u}_{3}-\mathrm{u}_{4}} & 0 & 0 & \frac{\mathrm{u}_{5}\left(\mathrm{u}_{3}-\mathrm{u}_{4}\right)}{\mathrm{u}_{1} \mathrm{u}_{3}-\mathrm{u}_{4}} \\
0 & 0 & \frac{\mathrm{u}_{4} \mathrm{u}_{6}-\mathrm{u}_{3} \mathrm{u}_{7}}{\mathrm{u}_{3}} & 0 \\
0 & 0 & 0 & 0
\end{array}\right)
$$

Now, let $\mathrm{V}^{[1]}=\left(\mathrm{v}_{1}^{[1]}, \mathrm{v}_{2}^{[1]}, \mathrm{v}_{3}^{[1]}, \mathrm{v}_{4}^{[1]}\right)^{\mathrm{T}}$ be the eigenvector corresponding to the eigenvalue $\lambda_{1 \mathrm{w}}=0$. Thus $\mathrm{J}_{1}^{*} \mathrm{~V}^{[1]}=0$, gives:

$\mathrm{V}^{[1]}=\left(\mathrm{v}_{1}^{[1]}, \frac{\mathrm{L}_{1}}{\mathrm{~L}_{2}} \mathrm{v}_{1}^{[1]}, 0, \frac{\mathrm{u}_{3}}{\mathrm{u}_{5}} \mathrm{v}_{1}^{[1]}\right)^{\mathrm{T}}$

Where, $\mathrm{L}_{1}=\mathrm{u}_{3}^{2}\left(\mathrm{u}_{1}\left(\mathrm{u}_{3}-\mathrm{u}_{5}\right)-\mathrm{u}_{4}+\mathrm{u}_{5}\right), \quad \mathrm{L}_{2}=\mathrm{u}_{5}\left(\mathrm{u}_{1} \mathrm{u}_{3}-\mathrm{u}_{4}\right)^{2}$ and $\mathrm{v}_{1}^{[1]}$ any nonzero real number. Clearly, $\mathrm{u}_{1} \mathrm{u}_{3} \neq \mathrm{u}_{4}$ due to the existence condition (3b). Let $\Psi^{[1]}=\left(\psi_{1}^{[1]}, \psi_{2}^{[1]}, \psi_{3}^{[1]}, \psi_{4}^{[1]}\right)^{\mathrm{T}}$ be the eigenvector associated with the eigenvalue $\lambda_{1 \mathrm{w}}=0$ of the matrix $\mathrm{J}_{1}^{* \mathrm{~T}}$. Then $\mathrm{J}_{1}^{* \mathrm{~T}} \Psi^{[1]}=0$, by solving this equation for $\Psi^{[1]}$ we get $\Psi^{[1]}=\left(0,0,0, \psi_{4}^{[1]}\right)^{\mathrm{T}}$, where $\psi_{4}^{[1]}$ any nonzero real number. Now, consider:

$\frac{\partial \mathrm{f}}{\partial \mathrm{u}_{11}}=\mathrm{f}_{\mathrm{u}_{11}}\left(\mathrm{X}, \mathrm{u}_{11}\right)=(0,0,0,-\mathrm{w})^{\mathrm{T}}$

Thus, $\mathrm{f}_{\mathrm{u}_{11}}\left(\mathrm{E}_{1}, \mathrm{u}_{11}^{*}\right)=(0,0,0,0)^{\mathrm{T}}$ and hence

$\left(\Psi^{[1]}\right)^{\mathrm{T}} \mathrm{f}_{\mathrm{u}_{11}}\left(\mathrm{E}_{1}, \mathrm{u}_{11}^{*}\right)=0$

So, according to Sotomayor's theorem the saddle-node bifurcation cannot occur, while the first condition of transcritical bifurcation is satisfied. Now, since:

$\operatorname{Df}_{\mathrm{u}_{11}}\left(\mathrm{X}, \mathrm{u}_{11}\right)=\left(\begin{array}{cccc}0 & 0 & 0 & 0 \\ 0 & 0 & 0 & 0 \\ 0 & 0 & 0 & 0 \\ 0 & 0 & 0 & -1\end{array}\right)$

Further, it is observed

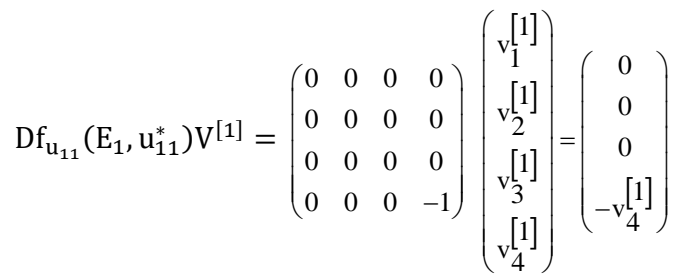

$$
\begin{aligned}
\left(\Psi^{[1]}\right)^{\mathrm{T}}\left[\mathrm{Df}_{\mathrm{u}_{11}}\left(\mathrm{E}_{1}, \mathrm{u}_{11}^{*}\right) \mathrm{V}^{[1]}\right] & =\left(0,0,0, \Psi_{4}^{[1]}\right)\left(0,0,0,-\mathrm{v}_{4}^{[1]}\right)^{\mathrm{T}} \\
& =-\mathrm{v}_{4}^{[1]} \Psi_{4}^{[1]} \neq 0
\end{aligned}
$$

Now, by substituting $\mathrm{V}^{[1]}$ in (24) we get

$D^{2} f\left(E_{1}, u_{11}^{*}\right)\left(V^{[1]}, V^{[1]}\right)$

$$
=\left(-2 \frac{L_{1}}{L_{2}}\left(v_{1}^{[1]}\right)^{2}, 0,0,2 \frac{s_{1} u_{3} L_{1}}{u_{5} L_{2}}\left(v_{1}^{[1]}\right)^{2}\right)^{T}
$$

Hence, it is obtained that:

$$
\begin{aligned}
\left(\Psi^{[1]}\right)^{\mathrm{T}}\left[D^{2} f\left(E_{1}, u_{11}^{*}\right)\left(V^{[1]}, V^{[1]}\right)\right] & =2 \frac{s_{1} u_{3} L_{1}}{u_{5} L_{2}}\left(v_{1}^{[1]}\right)^{2} \psi_{4}^{[1]} \\
& \neq 0
\end{aligned}
$$

Thus, according to Sotomayor's theorem system (2) has transcritical bifurcation at $E_{1}$ with the parameter $u_{11}=u_{11}^{*}$. Note that if the condition (25) does not satisfied then the system (2) does not have a bifurcation.

Theorem 11: Assume that the local stability condition (12c) hold and suppose that the condition

$\mathrm{u}_{2} \mathrm{u}_{8}+\mathrm{u}_{7} \neq \mathrm{u}_{2} \mathrm{u}_{6}+\mathrm{u}_{8}$

Is satisfied. Then when the parameter value $u_{11}$ passing through $u_{11}^{*}=\frac{u_{10}\left(u_{7}-u_{6}\right)}{u_{2} u_{6}-u_{7}}$ system (2) at the equilibrium point $E_{2}$ has:

1) No saddle-node bifurcation.

2) Transcritical bifurcation.

Proof: According to the Jacobian matrix $\mathrm{J}_{2}$ given by Eq. (12a) the system (2) at the equilibrium point $E_{2}$ has zero eigenvalue (say $\lambda_{2 \mathrm{w}}=0$ ) at $\mathrm{u}_{11}=\mathrm{u}_{11}^{*}$, and the Jacobian matrix $\mathrm{J}_{2}$ with $\mathrm{u}_{11}=\mathrm{u}_{11}^{*}$ becomes:

$\mathrm{J}_{2}^{*}=\mathrm{J}\left(\mathrm{u}_{11}=\mathrm{u}_{11}^{*}\right)$

$=\left(\begin{array}{cccc}\frac{u_{6}-u_{2} u_{6}}{u_{2} u_{6}-u_{7}} & \frac{u_{1} u_{6}-u_{7}}{u_{6}} & \frac{u_{2} u_{6}-u_{7}}{u_{6}} & 1 \\ 0 & \frac{u_{3} u_{7}-u_{4} u_{6}}{u_{6}} & 0 & 0 \\ \frac{u_{6}\left(u_{7}-u_{6}\right)}{u_{2} u_{6}-u_{7}} & 0 & 0 & \frac{u_{8}\left(u_{6}-u_{7}\right)}{u_{2} u_{6}-u_{7}} \\ 0 & 0 & 0 & 0\end{array}\right)$

Now, let $\mathrm{V}^{[2]}=\left(\mathrm{v}_{1}^{[2]}, \mathrm{v}_{2}^{[2]}, \mathrm{v}_{3}^{[2]}, \mathrm{v}_{4}^{[2]}\right)^{\mathrm{T}}$ be the eigenvector corresponding to the eigenvalue $\lambda_{2 \mathrm{w}}=0$. Thus $\mathrm{J}_{2}^{*} \mathrm{~V}^{[2]}=0$, gives:

$\mathrm{V}^{[2]}=\left(\mathrm{v}_{1}^{[2]}, 0, \frac{L_{3}}{L_{4}} \mathrm{v}_{1}^{[2]}, \frac{u_{6}}{u_{8}} \mathrm{v}_{1}^{[2]}\right)^{\mathrm{T}}$

Where $L_{3}=u_{6}^{2}\left(u_{2}\left(u_{8}-u_{6}\right)+u_{7}-u_{8}\right), L_{4}=u_{8}\left(u_{2} u_{6}-u_{7}\right)^{2}$ and $\mathrm{v}_{1}^{[2]}$ any nonzero real number. Clearly, $\mathrm{u}_{2} \mathrm{u}_{6} \neq \mathrm{u}_{7}$ due to the ex- 
istence condition (4b). Let $\Psi^{[2]}=\left(\psi_{1}^{[2]}, \psi_{2}^{[2]}, \psi_{3}^{[2]}, \psi_{4}^{[2]}\right)^{\mathrm{T}}$ be the eigenvector associated with the eigenvalue $\lambda_{2 \mathrm{w}}=0$ of the matrix $\mathrm{J}_{2}^{* \mathrm{~T}}$. Then $\mathrm{J}_{2}^{* \mathrm{~T}} \Psi^{[2]}=0$, by solving this equation for $\Psi^{[2]}$ we get $\Psi^{[2]}=\left(0,0,0, \Psi_{4}^{[2]}\right)^{\mathrm{T}}$, where $\psi_{4}^{[2]}$ any nonzero real number. Now, consider:

$\frac{\partial \mathrm{f}}{\partial \mathrm{u}_{11}}=\mathrm{f}_{\mathrm{u}_{11}}\left(\mathrm{X}, \mathrm{u}_{11}\right)=(0,0,0,-\mathrm{w})^{\mathrm{T}}$

Thus, $\mathrm{f}_{\mathrm{u}_{11}}\left(\mathrm{E}_{2}, \mathrm{u}_{11}^{*}\right)=(0,0,0,0)^{\mathrm{T}}$ and hence

$\left(\Psi^{[2]}\right)^{\mathrm{T}} \mathrm{f}_{\mathrm{u}_{11}}\left(\mathrm{E}_{2}, \mathrm{u}_{11}^{*}\right)=0$

So, according to Sotomayor's theorem the saddle-node bifurcation cannot occur, while the first condition of transcritical bifurcation is satisfied. Now, since:

$$
\mathrm{Df}_{\mathrm{u}_{11}}\left(\mathrm{X}, \mathrm{u}_{11}\right)=\left(\begin{array}{cccc}
0 & 0 & 0 & 0 \\
0 & 0 & 0 & 0 \\
0 & 0 & 0 & 0 \\
0 & 0 & 0 & -1
\end{array}\right)
$$

Therefore,

$$
\operatorname{Df}_{\mathrm{u}_{11}}\left(\mathrm{E}_{2}, \mathrm{u}_{11}^{*}\right) \mathrm{V}^{[2]}=\left(\begin{array}{cccc}
0 & 0 & 0 & 0 \\
0 & 0 & 0 & 0 \\
0 & 0 & 0 & 0 \\
0 & 0 & 0 & -1
\end{array}\right)\left(\begin{array}{c}
v_{1}^{[2]} \\
v_{2}^{[2]} \\
v_{3}^{[2]} \\
v_{4}^{[2]}
\end{array}\right)=\left(\begin{array}{c}
0 \\
0 \\
0 \\
-v_{4}^{[2]}
\end{array}\right)
$$

$\left(\Psi^{[2]}\right)^{\mathrm{T}}\left[\mathrm{Df}_{\mathrm{u}_{11}}\left(\mathrm{E}_{2}, \mathrm{u}_{11}^{*}\right) \mathrm{V}^{[2]}\right]=\left(0,0,0, \psi_{4}^{[2]}\right)\left(0,0,0,-\mathrm{v}_{4}^{[2]}\right)^{\mathrm{T}}$

$$
=-\mathrm{v}_{4}^{[2]} \psi_{4}^{[2]} \neq 0
$$

Now, by substituting $\mathrm{V}^{[2]}$ in (24) we get

$D^{2} f\left(E_{2}, u_{11}^{*}\right)\left(V^{[2]}, V^{[2]}\right)$

$$
=\left(-2 \frac{L_{3}}{L_{4}}\left(\mathrm{v}_{1}^{[2]}\right)^{2}, 0,0,2 \frac{u_{6} u_{10} L_{3}}{u_{8} L_{4}}\left(\mathrm{v}_{1}^{[2]}\right)^{2}\right)^{\mathrm{T}}
$$

Hence, it is obtained that:

$$
\begin{aligned}
\left(\Psi^{[2]}\right)^{\mathrm{T}}\left[\mathrm{D}^{2} \mathrm{f}\left(\mathrm{E}_{2}, \mathrm{u}_{11}^{*}\right)\right. & \left.\left(\mathrm{V}^{[2]}, \mathrm{V}^{[2]}\right)\right] \\
= & 2 \frac{u_{6} u_{10} L_{3}}{u_{8} L_{4}}\left(\mathrm{v}_{1}^{[2]}\right)^{2} \psi_{4}^{[2]} \neq 0
\end{aligned}
$$

Thus, according to Sotomayor's theorem system (2) has transcritical bifurcation at $\mathrm{E}_{2}$ with the parameter $\mathrm{u}_{11}=\mathrm{u}_{11}^{*}$.

\section{Theorem 12: Assume that the following conditions}

$$
\begin{aligned}
& u_{7}-u_{6} x_{3}>w_{3} \\
& u_{3} u_{8} \neq u_{5} u_{6} \\
& e_{13} e_{42} \neq e_{12} e_{43}
\end{aligned}
$$

Are satisfied. Then when the parameter value $u_{8}$ passing through $u_{8}^{*}=\frac{u_{7}-u_{6} x_{3}}{w_{3}}$ system (2) at the equilibrium point $E_{3}$ has:
1) No saddle-node bifurcation.

2) Transcritical bifurcation.

Proof: According to the Jacobian matrix $J_{3}$ given by Eq. (13a) the system (2) at the equilibrium point $E_{3}$ has zero eigenvalue (say $\lambda_{3 \mathrm{z}}=0$ ) at $\mathrm{u}_{8}=\mathrm{u}_{8}^{*}$, and the Jacobian matrix $\mathrm{J}_{3}$ with $\mathrm{u}_{8}=\mathrm{u}_{8}^{*}$ becomes:

$\mathrm{J}_{3}^{*}=\mathrm{J}\left(\mathrm{u}_{8}=\mathrm{u}_{8}^{*}\right)=\left(\mathrm{e}_{\mathrm{ij}}\right)_{4 \times 4}$

Where $e_{i j}=a_{i j}$ for all $\mathrm{i}, \mathrm{j}=1,2,3,4$ with $e_{33}=0$.

Let $\mathrm{V}^{[3]}=\left(\mathrm{v}_{1}^{[3]}, \mathrm{v}_{2}^{[3]}, \mathrm{v}_{3}^{[3]}, \mathrm{v}_{4}^{[3]}\right)^{\mathrm{T}}$ be the eigenvector corresponding to the eigenvalue $\lambda_{3 \mathrm{z}}=0$. Thus $\mathrm{J}_{3}^{*} \mathrm{~V}^{[3]}=0$, which gives:

$\mathrm{V}^{[3]}=\left(-\frac{e_{24} L_{5}}{L_{6}} \mathrm{v}_{3}^{[3]},-\frac{u_{10}}{s_{1}} \mathrm{v}_{3}^{[3]}, \mathrm{v}_{3}^{[3]}, \frac{e_{21} L_{5}}{L_{6}} \mathrm{v}_{3}^{[3]}\right)^{\mathrm{T}}$

Where $L_{5}=e_{13} e_{42}-e_{12} e_{43}, L_{6}=e_{42}\left(e_{11} e_{24}-e_{21}\right)$ and $\mathrm{v}_{3}^{[3]}$ any nonzero real number. Let $\Psi^{[3]}=\left(\psi_{1}^{[3]}, \psi_{2}^{[3]}, \psi_{3}^{[3]}, \psi_{4}^{[3]}\right)^{\mathrm{T}}$ be the eigenvector corresponding to the eigenvalue $\lambda_{3 z}=0$ of the matrix $J_{3}^{* T}$. Then we have $J_{3}^{* \mathrm{~T}} \Psi^{[3]}=0$, by solving this equation for $\Psi^{[3]}$ we get $\Psi^{[3]}=\left(0,0, \psi_{3}^{[3]}, 0\right)^{\mathrm{T}}$, where $\psi_{3}^{[3]}$ any nonzero real number. Now, consider:

$\frac{\partial \mathrm{f}}{\partial \mathrm{u}_{8}}=\mathrm{f}_{\mathrm{u}_{8}}\left(\mathrm{X}, \mathrm{u}_{8}\right)=(0,0,-\mathrm{zw}, 0)^{\mathrm{T}}$

Thus, $\mathrm{f}_{\mathrm{u}_{8}}\left(\mathrm{E}_{3}, \mathrm{u}_{8}^{*}\right)=(0,0,0,0)^{\mathrm{T}}$ and hence $\left(\Psi^{[3]}\right)^{\mathrm{T}} \mathrm{f}_{\mathrm{u}_{8}}\left(\mathrm{E}_{3}, \mathrm{u}_{8}^{*}\right)=0$

So, according to Sotomayor's theorem the saddle-node bifurcation cannot occur, while the first condition of transcritical bifurcation is satisfied. Now, since

$\mathrm{Df}_{\mathrm{u}_{8}}\left(\mathrm{X}, \mathrm{u}_{8}\right)=\left(\begin{array}{cccc}0 & 0 & 0 & 0 \\ 0 & 0 & 0 & 0 \\ 0 & 0 & -w & -z \\ 0 & 0 & 0 & 0\end{array}\right)$

Further, it is observed

$\mathrm{Df}_{\mathrm{u}_{8}}\left(\mathrm{E}_{3}, \mathrm{u}_{8}^{*}\right) \mathrm{V}^{[3]}=\left(\begin{array}{cccc}0 & 0 & 0 & 0 \\ 0 & 0 & 0 & 0 \\ 0 & 0 & -w & 0 \\ 0 & 0 & 0 & 0\end{array}\right)\left(\begin{array}{c}-\frac{e_{24} L_{5}}{L_{6}} v_{3}^{[3]} \\ -\frac{u_{10}}{s_{1}} v_{3}^{[3]} \\ v_{3}^{[3]} \\ \frac{e_{21} L_{5}}{L_{6}} v_{3}^{[3]}\end{array}\right)=\left(\begin{array}{c}0 \\ 0 \\ -w v_{3}^{[3]} \\ 0\end{array}\right)$

$\left(\Psi^{[3]}\right)^{\mathrm{T}}\left[\mathrm{Df}_{\mathrm{u}_{8}}\left(\mathrm{E}_{3}, \mathrm{u}_{8}^{*}\right) \mathrm{V}^{[3]}\right]=\left(0,0, \Psi_{3}^{[3]}, 0\right)\left(0,0,-w v_{3}^{[3]}, 0\right)^{\mathrm{T}}$

$$
=-w v_{3}^{[3]} \Psi_{3}^{[3]} \neq 0
$$

Now, by substituting $\mathrm{V}^{[3]}$ in (24) we get

$D^{2} f\left(E_{3}, u_{8}^{*}\right)\left(V^{[3]}, V^{[3]}\right)=\left(U_{1}, 0, U_{2}, 0\right)^{T}$

Where

$\mathrm{U}_{1}=2 \frac{e_{42} L_{5}\left(s_{1}-u_{10}\right)}{s_{1} L_{6}}\left(\mathrm{v}_{3}^{[3]}\right)^{2}$ 
$\mathrm{U}_{2}=-2 \frac{u_{11} L_{5}\left(u_{3} u_{8}-u_{5} u_{6}\right)}{s_{1} L_{6}}\left(\mathrm{v}_{3}^{[3]}\right)^{2}$

Hence, it is obtain that:

$\left(\Psi^{[3]}\right)^{T}\left[D^{2} f\left(E_{3}, u_{8}^{*}\right)\left(V^{[3]}, V^{[3]}\right)\right]=U_{2} \Psi_{3}^{[3]} \neq 0$

Thus, according to Sotomayor's theorem system (2) has transcritical bifurcation at $\mathrm{E}_{3}$ with the parameter $\mathrm{u}_{8}=\mathrm{u}_{8}^{*}$.

Theorem 13: Assume that the following conditions

$\mathrm{u}_{3} \mathrm{x}_{4}>\mathrm{u}_{5} \mathrm{w}_{4}$

$\mathrm{u}_{3} \mathrm{u}_{8} \neq \mathrm{u}_{5} \mathrm{u}_{6}$

$\mathrm{q}_{13} \mathrm{q}_{42} \neq \mathrm{q}_{12} \mathrm{q}_{43}$

Are satisfied. Then when the parameter value $u_{4}$ passing through $u_{4}^{*}=u_{3} x_{4}-u_{5} w_{4}$ system (2) at the equilibrium point $E_{4}$ has:

1) No saddle-node bifurcation.

2) Transcritical bifurcation.

Proof: According to the Jacobian matrix $\mathrm{J}_{4}$ given by Eq. (14a) the system (2) at the equilibrium $E_{4}$ has zero eigenvalue (say $\lambda_{4 y}=0$ ) at $\mathrm{u}_{4}=\mathrm{u}_{4}^{*}$, and the Jacobian matrix $\mathrm{J}_{4}$ with $\mathrm{u}_{4}=\mathrm{u}_{4}^{*}$ becomes:

$\mathrm{J}_{4}^{*}=\mathrm{J}\left(\mathrm{u}_{4}=\mathrm{u}_{4}^{*}\right)=\left(\mathrm{q}_{\mathrm{ij}}\right)_{4 \times 4}$

Where $q_{i j}=b_{i j}$ for all $\mathrm{i}, \mathrm{j}=1,2,3,4$ with $q_{22}=0$.

Let $\mathrm{V}^{[4]}=\left(\mathrm{v}_{1}^{[4]}, \mathrm{v}_{2}^{[4]}, \mathrm{v}_{3}^{[4]}, \mathrm{v}_{4}^{[4]}\right)^{\mathrm{T}}$ be the eigenvector corresponding to the eigenvalue $\lambda_{4 \mathrm{y}}=0$. Thus $\mathrm{J}_{4}^{*} \mathrm{~V}^{[4]}=0$, which gives:

$\mathrm{V}^{[4]}=\left(-\frac{q_{34} L_{7}}{L_{8}} \mathrm{v}_{2}^{[4]}, \mathrm{v}_{2}^{[4]},-\frac{u_{10}}{s_{1}} \mathrm{v}_{2}^{[4]}, \frac{q_{31} L_{7}}{L_{8}} \mathrm{v}_{2}^{[4]}\right)^{\mathrm{T}}$

Where $L_{7}=q_{12} q_{43}-q_{13} q_{42}, L_{8}=q_{43}\left(q_{11} q_{34}-q_{31}\right)$ and $\mathrm{v}_{2}^{[4]}$ any nonzero real number. Let $\Psi^{[4]}=\left(\psi_{1}^{[4]}, \psi_{2}^{[4]}, \psi_{3}^{[4]}, \psi_{4}^{[4]}\right)^{\mathrm{T}}$ be the eigenvector corresponding to the eigenvalue $\lambda_{4 y}=0$ of the matrix $\mathrm{J}_{4}^{* \mathrm{~T}}$. Then we have $\mathrm{J}_{4}^{* \mathrm{~T}} \Psi^{[4]}=0$, by solving this equation for $\Psi^{[4]}$ we get $\Psi^{[4]}=\left(0, \Psi_{2}^{[4]}, 0,0\right)^{\mathrm{T}}$, where $\psi_{2}^{[4]}$ any nonzero real number. Now, consider:

$\frac{\partial \mathrm{f}}{\partial \mathrm{u}_{4}}=\mathrm{f}_{\mathrm{u}_{4}}\left(\mathrm{X}, \mathrm{u}_{4}\right)=(0,-\mathrm{y}, 0,0)^{\mathrm{T}}$

Thus, $\mathrm{f}_{\mathrm{u}_{4}}\left(\mathrm{E}_{4}, \mathrm{u}_{4}^{*}\right)=(0,0,0,0)^{\mathrm{T}}$ and hence $\left(\Psi^{[4]}\right)^{\mathrm{T}} \mathrm{f}_{\mathrm{u}_{4}}\left(\mathrm{E}_{4}, \mathrm{u}_{4}^{*}\right)=0$. So, according to Sotomayor's theorem the saddle-node bifurcation cannot occur, while the first condition of transcritical bifurcation is satisfied. Now, since

$$
\operatorname{Df}_{\mathrm{u}_{4}}\left(X, \mathrm{u}_{4}\right)=\left(\begin{array}{cccc}
0 & 0 & 0 & 0 \\
0 & -1 & 0 & 0 \\
0 & 0 & 0 & 0 \\
0 & 0 & 0 & 0
\end{array}\right)
$$

Further, it is observed
$\mathrm{Df}_{\mathrm{u}_{4}}\left(\mathrm{E}_{4}, \mathrm{u}_{4}^{*}\right) \mathrm{V}^{[4]}=\left(\begin{array}{cccc}0 & 0 & 0 & 0 \\ 0 & -1 & 0 & 0 \\ 0 & 0 & 0 & 0 \\ 0 & 0 & 0 & 0\end{array}\right)\left(\begin{array}{c}-\frac{q_{34} L_{7}}{L_{8}} v_{2}^{[4]} \\ v_{2}^{[4]} \\ -\frac{u_{10}}{s_{1}} v_{2}^{[4]} \\ \frac{q_{31} L_{7}}{L_{8}} v_{2}^{[4]}\end{array}\right)=\left(\begin{array}{c}0 \\ -v_{2}^{[4]} \\ 0 \\ 0\end{array}\right)$

$\left(\Psi^{[4]}\right)^{\mathrm{T}}\left[\mathrm{Df}_{\mathrm{u}_{4}}\left(\mathrm{E}_{4}, \mathrm{u}_{4}^{*}\right) \mathrm{V}^{[4]}\right]=\left(0, \Psi_{2}^{[4]}, 0,0\right)\left(0,-v_{2}^{[4]}, 0,0\right)^{\mathrm{T}}$

$$
=-v_{2}^{[4]} \psi_{2}^{[4]} \neq 0
$$

Now, by substituting $\mathrm{V}^{[4]}$ in (24) we get

$D^{2} f\left(E_{4}, u_{4}^{*}\right)\left(V^{[4]}, V^{[4]}\right)=\left(U_{3}, U_{4}, 0, U_{5}\right)^{T}$

Where

$\mathrm{U}_{3}=2 \frac{q_{34} L_{7}\left(1-u_{10}\right)}{s_{1} L_{8}}\left(\mathrm{v}_{2}^{[4]}\right)^{2}$

$\mathrm{U}_{4}=-2 \frac{u_{11} L_{7}\left(u_{5} u_{6}-u_{3} u_{8}\right)}{u_{10} L_{8}}\left(\mathrm{v}_{2}^{[4]}\right)^{2}$

$\mathrm{U}_{5}=2 \frac{q_{31} L_{7}\left(s_{1}^{2}-u_{10}^{2}\right)}{s_{1} L_{8}}\left(\mathrm{v}_{2}^{[4]}\right)^{2}$

Hence, it is obtain that:

$\left(\Psi^{[4]}\right)^{\mathrm{T}}\left[\mathrm{D}^{2} \mathrm{f}\left(\mathrm{E}_{4}, \mathrm{u}_{4}^{*}\right)\left(\mathrm{V}^{[4]}, \mathrm{V}^{[4]}\right)\right]=\mathrm{U}_{4} \Psi_{2}^{[4]} \neq 0$

Thus, according to Sotomayor's theorem system (2) has transcritical bifurcation at $\mathrm{E}_{4}$ with the parameter $\mathrm{u}_{4}=\mathrm{u}_{4}^{*}$.

Remark: According to Sotomayor's theorem system (2) has no bifurcation at the nonhyperbolic equilibrium point $E_{5}$ for different parameter values, and that ensure the nonhyperbolic is a necessary but not sufficient condition for bifurcation to occur.

Theorem14: system (2) has no bifurcation at equilibrium point $E_{6}$. Proof: According to the Jacobian matrix $\mathrm{J}_{6}$ given by Eq. (16.a) the system (2) at the equilibriumpoint $E_{4}$ has zero eigenvalue (say $\left.\lambda_{6}=0\right)$ at $\mathrm{u}_{6}=\mathrm{u}_{6}^{*}$ and the Jacobian matrix $\mathrm{J}_{6}$ with $\mathrm{u}_{6}=\mathrm{u}_{6}^{*}$ bcomes:

$\mathrm{J}_{6}^{*}=\mathrm{J}\left(\mathrm{u}_{6}=\mathrm{u}_{6}^{*}\right)=\left(\mathrm{h}_{\mathrm{ij}}\right)_{4 \times 4}$

Where $h_{i j}=d_{i j}$ for all $\mathrm{i}, \mathrm{j}=1,2,3,4$ with $h_{31}=\mathrm{u}_{6}^{*} \mathrm{z}$.

Let $\mathrm{V}^{[6]}=\left(\mathrm{v}_{1}^{[6]}, \mathrm{v}_{2}^{[6]}, \mathrm{v}_{3}^{[6]}, \mathrm{v}_{4}^{[6]}\right)^{\mathrm{T}}$ be the eigenvector corresponding to the eigenvalue $\lambda_{6}=0$. Thus $J_{6}^{*} V^{[6]}=0$, which gives:

$\mathrm{V}^{[6]}=\left(\frac{u_{5}}{u_{3}} \mathrm{v}_{4}^{[6]},-\frac{\left(h_{43} L_{9}+L_{10}\right)}{L_{11}} \mathrm{v}_{4}^{[6]},-\frac{\left(h_{42} L_{9}+L_{10}\right)}{L_{11}} \mathrm{v}_{4}^{[6]}, \mathrm{v}_{4}^{[6]}\right)^{\mathrm{T}}$

Where $\quad L_{9}=h_{24} h_{11}-h_{21} \quad, \quad L_{10}=h_{44} h_{12} h_{21}$ $L_{11}=h_{21}\left(h_{12} h_{43}-h_{13} h_{42}\right)$ and $\mathrm{v}_{4}^{[6]}$ any nonzero real number.

Let $\Psi^{[6]}=\left(\psi_{1}^{[6]}, \psi_{2}^{[6]}, \psi_{3}^{[6]}, \psi_{4}^{[6]}\right)^{\mathrm{T}}$ be the eigenvector corresponding to the eigenvalue $\lambda_{6}=0$ of the matrix $\mathrm{J}_{6}^{* \mathrm{~T}}$. Then we have $\mathrm{J}_{6}^{* \mathrm{~T}} \Psi^{[6]}=0$, by solving this equation for $\Psi^{[6]}$ we get : 
$\Psi^{[6]}=\left(0,-\frac{h_{34}}{h_{24}} \psi_{3}^{[6]}, \Psi_{3}^{[6]}, 0\right)^{\mathrm{T}}$

Where $\psi_{3}^{[6]}$ any nonzero real number. Now, consider:

$\frac{\partial \mathrm{f}}{\partial \mathrm{u}_{6}}=\mathrm{f}_{\mathrm{u}_{6}}\left(\mathrm{X}, \mathrm{u}_{6}\right)=(0,0, \mathrm{xz}, 0)^{\mathrm{T}}$

Thus, $\mathrm{f}_{\mathrm{u}_{6}}\left(\mathrm{E}_{6}, \mathrm{u}_{6}^{*}\right)=(0,0, \mathrm{xz}, 0)^{\mathrm{T}}$ and hence

$\left(\Psi^{[6]}\right)^{\mathrm{T}} \mathrm{f}_{\mathrm{u}_{6}}\left(\mathrm{E}_{6}, \mathrm{u}_{6}^{*}\right)=\mathrm{xz} \Psi_{3}^{[6]}$.

Now, by substituting $\mathrm{V}^{[6]}$ in (24) we get

$D^{2} f\left(E_{6}, u_{6}^{*}\right)\left(V^{[6]}, V^{[6]}\right)=\left(U_{6}, 0,0, U_{7}\right)^{T}$

Where

$\mathrm{U}_{6}=-2 \frac{u_{5}\left(2 L_{10}+\left(h_{42}+h_{43}\right) L_{9}\right)}{u_{3} L_{11}}\left(\mathrm{v}_{4}^{[6]}\right)^{2}$

$\mathrm{U}_{7}=-2 \frac{s_{1}\left(h_{43} L_{9}+L_{10}\right)+u_{10}\left(h_{42} L_{9}+L_{10}\right)}{L_{11}}\left(\mathrm{v}_{4}^{[6]}\right)^{2}$

Hence, it is obtain that:

$\left(\Psi^{[6]}\right)^{T}\left[D^{2} f\left(E_{6}, u_{6}^{*}\right)\left(V^{[6]}, V^{[6]}\right)\right]=0$

Thus, according to Sotomayor's theorem system (2) has no bifurcation at $\mathrm{E}_{6}$ with the parameter $\mathrm{u}_{6}=\mathrm{u}_{6}^{*}$.

\section{The Hopf bifurcation analysis}

In this section, the occurrence of Hopf bifurcation of system (2) near the positive equilibrium point $\mathrm{E}_{6}$ is studied below.

Theorem 15: Assume that the following conditions are hold:

$\Gamma_{1}>\frac{d_{11}^{2} d_{44} d_{43}}{d_{21} \Gamma_{6}}$

$\Gamma_{2}>d_{11}\left(d_{11} d_{34} d_{43}+d_{44} \Gamma_{2}\right)+d_{44}\left(d_{44} d_{34}-d_{11} \Gamma_{1}+\Gamma_{4}\right)$

Then system (2) possesses a Hopf bifurcation around the equilibrium point $E_{6}$ when the parameter $u_{5}$ passes through $u_{5}=u_{5}^{*}$, where

$$
u_{5}^{*}=\frac{1}{2 y_{6} R_{1}}\left(-R_{2}+\sqrt{R_{2}^{2}-4 R_{1} R_{3}}\right)
$$

With

$R_{1}=d_{11} d_{44} d_{42}$

$R_{2}=d_{31} \Gamma_{1}^{2} \Gamma_{6}+d_{11} d_{44} d_{42} \Gamma_{1}\left(d_{11}+d_{44} \Gamma_{4}\right)-2 d_{11} d_{44} d_{42} d_{34} d_{43}$

$$
-d_{42}\left(d_{11} \Gamma_{4}+d_{44} \Gamma_{2}\right)
$$

$R_{3}=\Gamma_{2}\left[d_{11}\left(d_{11} d_{34} d_{43}+d_{44} \Gamma_{2}\right)+d_{44}\left(d_{44} d_{34} d_{43}-d_{11} \Gamma_{1}+\Gamma_{4}\right)\right]$

$-\Gamma_{1} \Gamma_{4}\left[d_{11} d_{44}\left(\Gamma_{2}+d_{44} d_{34} d_{43}+\Gamma_{4}\right)\right]$

$+\Gamma_{1}\left[d_{34}\left(d_{21} \Gamma_{1} \Gamma_{6}-d_{11} d_{44} d_{43}\right)\right]$

Proof: According to the Hopf bifurcation theorem, the Hopf bifurcation can occur provided that: $D_{i}\left(u_{5}^{*}\right)>0, \mathrm{i}=1,3 ; \Delta_{1}>0$,
$D_{1}^{3}-4 \Delta_{1}>0$ and $\Delta_{2}\left(u_{5}^{*}\right)=0$. Therefore we obtain that $\Delta_{2}=0$ gives

$u_{5}^{2} y_{6}^{2} R_{1}+u_{5} y_{6} R_{2}+R_{3}=0$

Then Eq. (29c) has a unique positive root

$u_{5}^{*}=\frac{1}{2 y_{6} R_{1}}\left(-R_{2}+\sqrt{R_{2}^{2}-4 R_{1} R_{3}}\right)$

Provided that the conditions (29)(a,b) hold. Now, at $u_{5}=u_{5}^{*}$ the characteristic equation can be written as:

$\left(\lambda_{6}^{2}+\frac{D_{3}}{D_{1}}\right)\left(\lambda_{6}^{2}+D_{1} \lambda_{6}+\frac{\Delta_{1}}{D_{1}}\right)=0$

Which has four roots

$\lambda_{61,2}= \pm i \sqrt{\frac{D_{3}}{D_{1}}}, \lambda_{63,4}=\frac{1}{2}\left(-D_{1} \pm \sqrt{D_{1}^{2}-4 \frac{\Delta_{1}}{D_{1}}}\right)$

Clearly, at $u_{5}=u_{5}^{*}$ there are two pure imaginary eigenvalues $\left(\lambda_{61,2}\right.$ and $\left.\lambda_{63,4}\right)$ and two eigenvalues which are real and negative. Now, for all values of $u_{5}$ in the neighborhood of $u_{5}^{*}$, the roots in general of the following form:

$\lambda_{61}=\alpha_{1}+i \alpha_{2} ; \lambda_{62}=\alpha_{1}-i \alpha_{2} ; \lambda_{63,4}=\frac{1}{2}\left(-D_{1} \pm \sqrt{D_{1}^{2}-4 \frac{\Delta_{1}}{D_{1}}}\right)$

Clearly, $\left.\operatorname{Re}\left(\lambda_{6 k}\left(u_{5}\right)\right)\right|_{u_{5}=u_{5}^{*}}=\alpha_{1}\left(u_{5}^{*}\right)=0, k=1,2$

That means the first condition of necessary and sufficient conditions for Hopf bifurcation is satisfied at $u_{5}=u_{5}^{*}$. Now, to verify the transversality condition we must prove that $\bar{\Theta}\left(u_{5}^{*}\right) \bar{\Psi}\left(u_{5}^{*}\right)+\bar{\Gamma}\left(u_{5}^{*}\right) \bar{\Phi}\left(u_{5}^{*}\right) \neq 0$, Note that for $u_{5}=u_{5}^{*}$ we have:

$\alpha_{1}=0, \quad \alpha_{2}=\sqrt{\frac{D_{3}}{D_{1}}}$

Then

$$
\begin{aligned}
& \bar{\Psi}\left(u_{5}^{*}\right)=-2 D_{3}\left(u_{5}^{*}\right) \\
& \bar{\Phi}\left(u_{5}^{*}\right)=2 \frac{\alpha_{2}\left(u_{5}^{*}\right)}{D_{1}}\left(D_{1} D_{2}-2 D_{3}\right) \\
& \bar{\Theta}\left(u_{5}^{*}\right)=D_{4}^{\prime}\left(u_{5}^{*}\right)-\frac{D_{3}\left(u_{5}^{*}\right)}{D_{1}\left(u_{5}^{*}\right)} D_{2}^{\prime}\left(u_{5}^{*}\right) \\
& \bar{\Gamma}\left(u_{5}^{*}\right)=\alpha_{2}\left(u_{5}^{*}\right)\left(D_{3}^{\prime}\left(u_{5}^{*}\right)-\frac{D_{3}\left(u_{5}^{*}\right)}{D_{1}\left(u_{5}^{*}\right)} D_{1}^{\prime}\left(u_{5}^{*}\right)\right)
\end{aligned}
$$

Where:

$$
\begin{aligned}
& D_{1}^{\prime}=\left.\frac{d D_{1}}{d u_{5}}\right|_{u_{5}=u_{5}^{\prime}}=0 \\
& D_{2}^{\prime}=\left.\frac{d D_{2}}{d u_{5}}\right|_{u_{5}=u_{5}^{*}}=-y_{6} d_{42} \\
& D_{3}^{\prime}=\left.\frac{d D_{3}}{d u_{5}}\right|_{u_{5}=u_{5}^{*}}=y_{6} d_{11} d_{42} \\
& D_{4}^{\prime}=\left.\frac{d D_{4}}{d u_{5}}\right|_{u_{5}=u_{5}^{\prime \prime}}=y_{6} d_{31} \Gamma_{6}
\end{aligned}
$$


Then we have

$$
\begin{aligned}
& \bar{\Theta}\left(u_{5}^{*}\right) \bar{\Psi}\left(u_{5}^{*}\right)+\bar{\Gamma}\left(u_{5}^{*}\right) \bar{\Phi}\left(u_{5}^{*}\right)= \\
& 2 y_{6} D_{1} D_{3}\left[d_{42}\left(d_{11}\left(D_{2}-2 \frac{D_{3}}{D_{1}}\right)-D_{1} D_{3}\right)-d_{31} \Gamma_{6}\right] \neq 0
\end{aligned}
$$

So, we obtain that the Hopf bifurcation occurs around the equilibrium point $\mathbf{E}_{\mathbf{6}}$ at the parameter $\mathbf{u}_{\mathbf{5}}=\mathbf{u}_{\mathbf{5}}^{*}$.

\section{Numerical analysis}

In this section the dynamical behavior of system (2) is studied numerically starting at different sets of initial points with different sets of parameters values. The objectives of this study are: first specify the control parameters on the dynamical behavior of system (2) and second ensure our obtained analytical results. It is observed that, for the following set of hypothetical parameters:

$\mathrm{u}_{1}=0.1, \mathrm{u}_{3}=0.5, \mathrm{u}_{4}=0.1, \mathrm{u}_{5}=0.4, \mathrm{u}_{6}=0.5$,

$\mathrm{u}_{7}=0.1, \mathrm{u}_{8}=0.4, \mathrm{u}_{10}=0.2, \mathrm{u}_{11}=0.2, \mathrm{~s}_{1}=0.2$

The solution of system (2) approaches asymptotically to the positive equilibrium point $\mathrm{E}_{6}=(1.13,0.46,0.53,1.16)$ and this is confirming our obtained analytical results as shown in Fig. (1).

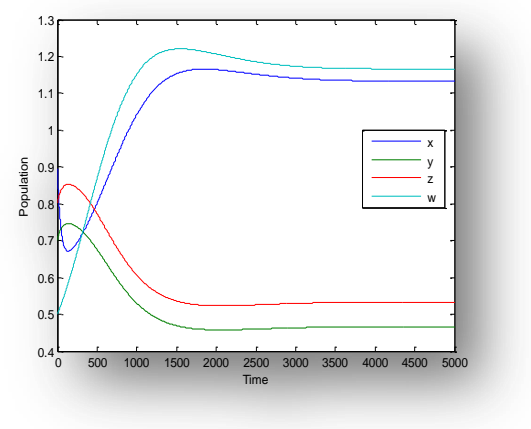

Fig. 1: Time series of the solution of system (2) that approaches asymptoticallyto the stable positive equilibrium point $(1.13,0.46,0.53,1.16)$

Now, in order to specify the control parameters values of system (2), the system is solved numerically for the data given in (29) with varying one parameter each time. It is observed that, for the data given in (29) with varying one of the parameter values $\mathrm{u}_{1}, \mathrm{u}_{2}$, $\mathrm{u}_{10}$ and $\mathrm{u}_{11}$, there is no change in the dynamical behavior of system (2) and the system still approaches to the positive equilibrium point and hence these parameters are not control parameters. It is observed that for the data as given in (29) with $u_{3}<0.5$, the solution of system (2) approaches asymptotically to $E_{4}$ as shown in Fig. (2a), however for $u_{3}>0.5$, the solution of system (2) approaches asymptotically to $E_{3}$ as shown in Fig. (2b). The solution of system (2) has similar behavior as that of varying $u_{3}$ when $u_{8}$ passing through 0.4 .
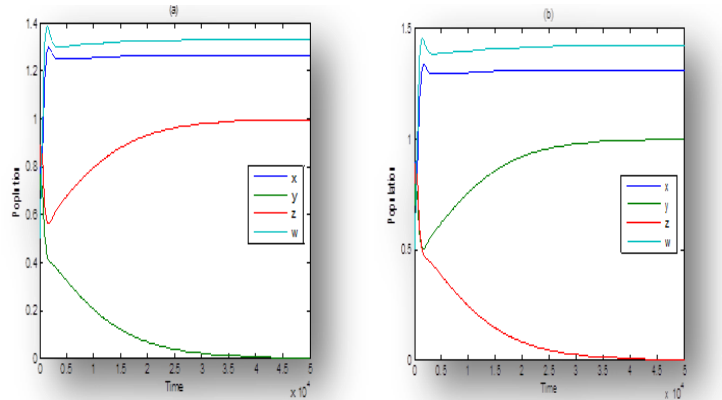

Fig. 2: Time series of the solution of system (2) for the data given by (29) with(a) $\mathrm{u}_{3}=0.49$, which approaches to $(1.26,0,0.99,1.33)$ in the interior of positive octant of $x z w-s p a c e,(b) \mathrm{u}_{3}=0.51$, which approaches to (1.31, $0.99,0,1.42)$ in the interior of positive octant of $x y w$-space

For the data given in (29) with $\mathrm{u}_{5}<0.4$, the solution of system (2) approaches asymptotically to $E_{3}$ as shown in Fig. (3a), however for $\mathrm{u}_{5}>0.4$, the solution of system (2) approaches asymptotically to $E_{4}$ as shown in Fig. (3b). the solution of system (2) has similar behavior as that of varying $\mathrm{u}_{5}$ when $\mathrm{u}_{6}$ passing through 0.5 .
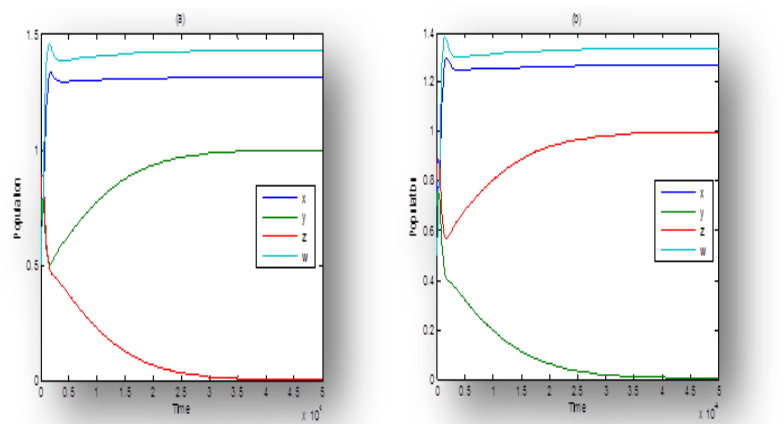

Fig. 3: Time series of the solution of system (2) for the data given by (29) with (a) $\mathrm{u}_{5}=0.39$, which approaches to $(1.31,0.99,0,1.42)$ in the interior of positive octant of $x y w$-space, (b) $u_{5}=0.41$, which approaches to $(1.26,0$ $0.99,1.33)$ in the interior of positive octant of $x z w$-space

For the data given in (29) with $\mathrm{u}_{4}>0.1$, the solution of system (2) approaches asymptotically to $\mathrm{E}_{4}$ as shown in Fig. (4a). However, for $\mathrm{u}_{7}>0.1$ with the rest of parameters as given in (29), the solution of system (2) approaches asymptotically to $E_{3}$ as shown in Fig. (4b).
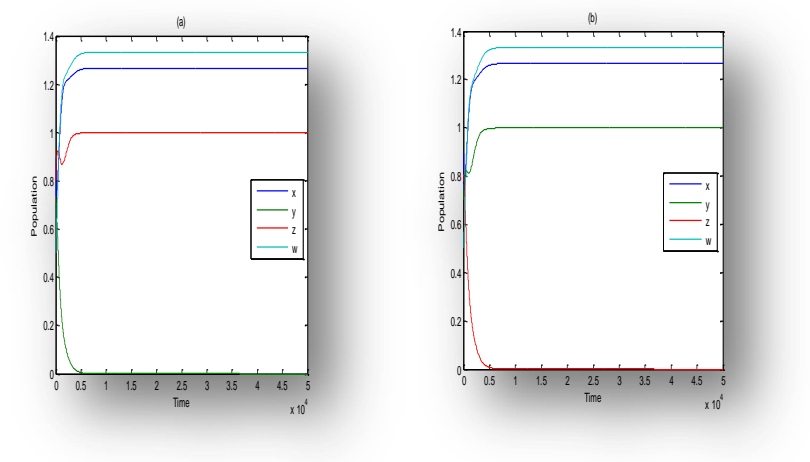

Fig. 4: Time series of the solution of system (2) for the data given by (29) with (a) $\mathrm{u}_{4}=0.2$, which approaches to $(1.26,0,1,1.33)$ in the interior of positive octant of $x z w$-space, (b) $\mathrm{u}_{7}=0.2$, which approaches to $(1.26,1,0$, 1.33 ) in the interior of positive octant of $x y w$-space

For the data give $n$ in (29) with $s_{1} \leq-0.22$, the solution of system (2) approaches asymptotically to $\mathrm{E}_{5}$ as shown in Fig. (5). 


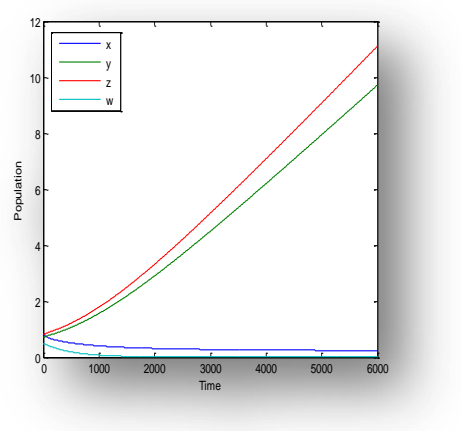

Fig. 5: Time series of the solution of system (2) for the data given by (29) with $\mathrm{s}_{1}=-0.22$, which approaches to $(0.23,9.7,11.09,0)$

Now by varying the parameters $\mathrm{u}_{4}, \mathrm{u}_{7}$ and $\mathrm{s}_{1}$ keeping the rest of parameters values as in (29), it is observed that for $u_{4}>0.5$, $\mathrm{u}_{7}>0.5$ and $\mathrm{s}_{1} \leq-0.22$, the solution of system (2) approaches asymptotically to $E_{0}$ as shown in Fig. (6a). However for $\mathrm{u}_{4}<0.5$, $\mathrm{u}_{7}<0.5$ and $\mathrm{s}_{1} \leq-0.22$, the solution of system (2) approaches asymptotically to $E_{1}$ as shown in Fig.(6b). Moreover for $\mathrm{u}_{4}>0.5$, $\mathrm{u}_{7}<0.5$ and $\mathrm{s}_{1} \leq-0.22$, the solution of system (2) approaches asymptotically to $E_{2}$ as shown in Fig. (6c).
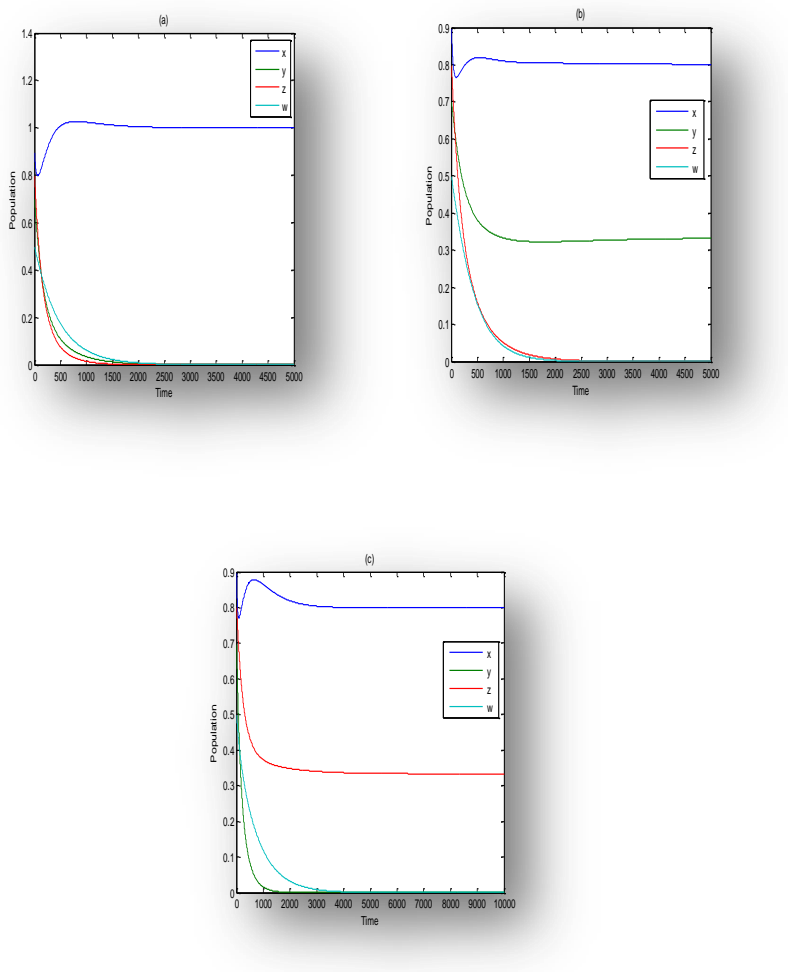

Fig. 6: Time series of the solution of system (2) for the data given by (29) with (a) $\mathrm{u}_{4}=0.7, \mathrm{u}_{7}=0.8$ and $\mathrm{s}_{1}=-0.23$, which approaches to $(1,0,0,0)$ on the $x$-axis, (b) $\mathrm{u}_{4}=0.4, \mathrm{u}_{7}=0.6$ and $\mathrm{s}_{1}=-0.22$, which approaches to $(0.8,0.3,0,0)$ in Int. $R_{+}^{2}$ of $x y$-plane, $(c) \mathrm{u}_{4}=0.7, \mathrm{u}_{7}=0.4$ and $\mathrm{s}_{1}=-0.23$, which approaches to $(0.7,0,0.3,0)$ in Int. $\mathrm{R}_{+}^{2}$ of $x z$-plane

\section{Conclusion}

In the previous section, we proposed and analyzed an ecological model that described the dynamical behavior of food web model with Lotka-Volterra type of functional response. It is assumed that: The phytoplankton divided into two compartments namely toxin producing phytoplankton which produces a toxic substance as a defensive strategy against predation by zooplankton, and a nontoxic phytoplankton. However the portion of the dead species of phytoplankton and zooplankton is returned to nutrient due to the decomposition operation. The boundedness of the proposed sys- tem (2) has been discussed. The dynamical behavior of system (2) has been investigated locally as well as globally. To understand the effect of varying each parameter on the dynamics of system (2) and to confirm our obtained analytical results, system (2) has been solved numerically for a biological feasible set of hypothetical parameters values and the following results are obtained:

1) For the set of hypothetical parameters values given by (29) system (2) approaches asymptotically to stable positive equilibrium point, and hence the food web system coexists (persist).

2) It is observed that varying the parameters: $u_{1}, u_{2}$ which stand for conversion rate from death (toxic, nontoxic) phytoplankton to nutrient, the consumption rate from nontoxic phytoplankton to zooplankton $\mathrm{u}_{10}$ and the zooplankton natural death rate $u_{11}$, do not have any effect on the dynamical behavior of system (2) and the system still approaches to a positive equilibrium point.

3) As the consumption rates from nutrient to toxic phytoplankton $u_{3}$ decreases from a critical value $(0.5)$ keeping other parameters fixed as in (29) then the toxic phytoplankton faces extinction and the solution of system (2) approaches asymptotically to equilibrium point $\mathrm{E}_{4}$ in the Int. $\mathrm{R}_{+}^{3}$ of $\mathrm{xzw}$-space. While increasing $\mathrm{u}_{3}$ from that critical value will causes extinction in the nontoxic phytoplankton species and the solution of system (2) approaches asymptotically to equilibrium point $E_{3}$ in the Int. $R_{+}^{3}$ of xyw-space. It is observed that the consumption rate $\mathrm{u}_{8}$ has the same effect as $\mathrm{u}_{3}$ with different critical value. Clearly, these critical values are bifurcation points.

4) As the consumption rate from toxic phytoplankton to zooplankton $u_{5}$ decreases from a critical value (0.4) keeping other parameters fixed as in (29) then the nontoxic phytoplankton faces extinction and the solution of system (2) approaches asymptotically to equilibrium point $E_{3}$ in the Int. $\mathrm{R}_{+}^{3}$ of xyw-space. While increasing $\mathrm{u}_{5}$ from that critical value will causes extinction in the toxic phytoplankton species and the solution of system (2) approaches asymptotically to equilibrium point $\mathrm{E}_{4}$ in the Int. $\mathrm{R}_{+}^{3}$ of $\mathrm{xzw}$-space. It is observed that the consumption rate $\mathrm{u}_{6}$ has the same effect as $\mathrm{u}_{5}$ with different critical value. Clearly, these critical values are bifurcation points.

5) As the toxic phytoplankton natural death rate $u_{4}$ increases from acritical value (0.1) keeping other parameters fixed as in (29) then again the toxic phytoplankton faces extinction and the solution of system (2) approaches asymptotically to equilibrium point $\mathrm{E}_{4}$, that means the system losses the persistence. Otherwise the solution still approaches to the positive equilibrium point. However, increasing nontoxic phytoplankton natural death rate $\mathrm{u}_{7}$ from the same critical value with the other parameters as given in (29) has extinction effect in the nontoxic phytoplankton and the system approaches asymptotically to $E_{3}$ again that means the system losses the persistence. Otherwise the solution still approaches to the positive equilibrium point. Finally, these critical values represent bifurcation points.

6) Gradually decreasing the parameter $s_{1}$ from the critical value $(-0.23)$ which stand for the difference between the consumption rate from toxic phytoplankton and the liberation rate of toxin substance, causes extinction in the zooplankton species and the system approaches to $E_{5}$ in the Int. $\mathrm{R}_{+}^{3}$ of xyz-space. Hence, the system (2) bifurcate at that critical point.

7) As increasing the parameters $\mathrm{u}_{4}, \mathrm{u}_{7}$ with $\mathrm{s}_{1} \leq-0.22$ causes extinction effect in phytoplankton (toxic, nontoxic) and zooplankton and the system approaches to $E_{0}$ on the $\mathrm{x}-$ axis. However decreasing the value of $\mathrm{u}_{4}$ and increasing $\mathrm{u}_{7}$ with $s_{1} \leq-0.22$ causes extinction effect in the nontoxic phytoplankton and zooplankton and the system approaches to $E_{1}$ on $x y-$ plane. While increasing the value of $u_{4}$ and decreasing $u_{7}$ with $s_{1} \leq-0.22$ causes extinction effect in the 
toxic phytoplankton and zooplankton and the system approaches to $\mathrm{E}_{2}$ on $\mathrm{xz}$ - plane.

\section{Acknowledgement}

All thanks to Professor Dr. Raid Kamel Naji for very helpful suggestions, who gave me a lot of his time, and effort, guidance, and his sound opinions. I also wish to thank Dr. Azhar Abbas Majeed, for her guidance and encouragement during the supervision of my work, who I stand her all the respect and Allah bless her and extension in life and grant health and safety to be an asset to the science. Also I express my deep gratitude to college of science and colleagues who provided help me to accomplish this paper wishing to be modest effort can benefit from the service of science.

\section{References}

[1] P.J.S. Franks, "NPZ models of plankton dynamics: their construction, coupling to physics, and application," Journal of Oceanography, vol. 58, pp. 379-387, (2002) http://dx.doi.org/10.1023/A:1015874028196.

[2] S.R.J. Jang, J. Baglama and J. Rick, "Nutrient-phytoplanktonzooplankton models with a toxin," Mathematical and Computer Modelling, vol. 43, no. 1-2, pp. 105-118, (2006). http://dx.doi.org/10.1016/j.mcm.2005.09.030.

[3] B. Mukhopadhyay and R. Bhattacharyya, "Modelling phytoplankton allelopathy in a nutrient-plankton model with spatial heterogeneity," Ecological Modelling, vol. 198, no. 1-2, pp. 163-173, (2006). http://dx.doi.org/10.1016/j.ecolmodel.2006.04.005.

[4] S. Roy, "The coevolution of two phytoplankton species on a single resource: allelopathy as a pseudo-mixotrophy," Theoretical Population Biology, vol. 75, no. 1, pp. 68-75, (2009) http://dx.doi.org/10.1016/j.tpb.2008.11.003.

[5] T. Saha and M. Bandyopadhyay, "Dynamical analysis of toxin producing phytoplankton-zooplankton interactions," Nonlinear Analysis: Real World Applications, vol. 10, no. 1, pp. 314-332, (2009). http://dx.doi.org/10.1016/j.nonrwa.2007.09.001.

[6] S. Chakraborty, S. Roy and J. Chattopadhyay, "Nutrient-limited toxin production and the dynamics of two phytoplankton in culture media: A mathematical model," ecological modeling, vol. 213, pp. 191-201, (2008).

[7] A. Fan, P. Han and K. Wang, "Global dynamics of a nutrientplankton system in the water ecosystem," Applied Mathematics and Computation, vol. 219, pp. 8269-8276, (2013). http://dx.doi.org/10.1016/j.amc.2013.02.051.

[8] M.G. Danielsdottir, M.T. Brett and G.B. Arhonditsis, "Phytoplankton food quality control of planktonic food web processes," Hydrobiologia, vol. 589, pp. 29-41, (2007). http://dx.doi.org/10.1007/s10750-007-0714-6.

[9] R.K. Upadhyay and J. Chattopadhyay, "Chaos to order: role of toxin producing phytoplankton in aquatic systems," Nonlinear Analysis. Modelling and Control, vol. 10, no. 4, pp. 383-396, (2005).

[10] K. Das and S. Ray, "Effect of delay on nutrient cycling in phytoplankton-zooplankton interactions in estuarine system," Ecological Modelling, vol. 215, no. 1-3, pp. 69-76, (2008). http://dx.doi.org/10.1016/j.ecolmodel.2008.02.019

[11] R.R. Sarkar, S. Pal and J. Chattopadhyay, "Role of two toxinproducing plankton and their effect on phytoplankton-zooplankton system-a mathematical study supported by experimental findings," Bio System, vol. 80, pp.11-23, (2005) http://dx.doi.org/10.1016/j.biosystems.2004.09.029.

[12] C.M. Lalli and T.R. Parsons, "Biological Oceanograph : Butterworth-Heinemann," (1997).

[13] S.J. Rashid and R.K. Naji, "Stability and bifurcation of aquatic food chain model," Mathematical Theory and Modeling, vol. 4, no. 14, (2014).

[14] L. Perko, "Differential Equation and Dynamical Systems," third Edition, New York, Springer, (2001). http://dx.doi.org/10.1007/978-1-4613-0003-8. 